\title{
Existence Results for Langevin Fractional Differential Inclusions Involving Two Fractional Orders with Four-Point Multiterm Fractional Integral Boundary Conditions
}

\author{
Ahmed Alsaedi, ${ }^{1}$ Sotiris K. Ntouyas, ${ }^{2}$ and Bashir Ahmad ${ }^{1}$ \\ ${ }^{1}$ Department of Mathematics, Faculty of Science, King Abdulaziz University, P.O. Box 80203, Jeddah 21589, Saudi Arabia \\ ${ }^{2}$ Department of Mathematics, University of Ioannina, 45110 Ioannina, Greece
}

Correspondence should be addressed to Ahmed Alsaedi; aalsaedi@hotmail.com

Received 2 January 2013; Accepted 22 March 2013

Academic Editor: Juan J. Nieto

Copyright (C) 2013 Ahmed Alsaedi et al. This is an open access article distributed under the Creative Commons Attribution License, which permits unrestricted use, distribution, and reproduction in any medium, provided the original work is properly cited.

We discuss the existence of solutions for Langevin fractional differential inclusions involving two fractional orders with four-point multiterm fractional integral boundary conditions. Our study relies on standard fixed point theorems for multivalued maps and covers the cases when the right-hand side of the inclusion has convex as well as nonconvex values. Illustrative examples are also presented.

\section{Introduction}

We consider a boundary value problem of nonlinear Langevin fractional differential inclusions involving two fractional orders with four-point multiterm fractional integral boundary conditions given by

$$
\begin{gathered}
{ }^{c} D^{p}\left({ }^{c} D^{q}+\lambda\right) x(t) \in F(t, x(t)), \quad 0<t<1, \\
x(0)=\sum_{i=1}^{n} \beta_{i}\left(I^{\mu_{i}} x\right)(\zeta), \\
x(1)=\sum_{i=1}^{n} \alpha_{i}\left(I^{v_{i}} x\right)(\eta), \quad 0<\zeta<\eta<1,
\end{gathered}
$$

where $0<p, q \leq 1,{ }^{c} D^{q}$ denotes the Caputo fractional derivative of order $q, \lambda$ is a real number, $I^{\kappa}$ is the RiemannLiouville fractional integral of order $\kappa>0\left(\kappa=v_{i}, \mu_{i} ; i=\right.$ $1,2, \ldots, n, n \in \mathbb{N}=\{1,2, \ldots\})$, and $\alpha_{i}, \beta_{i} \in \mathbb{R}$ are suitably chosen constants.

In recent years, the boundary value problems of fractional order differential equations have emerged as an important area of research, since these problems have applications in various disciplines of science and engineering such as mechanics, electricity, chemistry, biology, economics, control theory, signal and image processing, polymer rheology, regular variation in thermodynamics, biophysics, aerodynamics, viscoelasticity and damping, electrodynamics of complex medium, wave propagation, and blood flow phenomena [1-5]. Many researchers have studied the existence theory for nonlinear fractional differential equations with a variety of boundary conditions; for instance, see the papers [6-17] and the references therein.

The Langevin equation (first formulated by Langevin in 1908 ) is found to be an effective tool to describe the evolution of physical phenomena in fluctuating environments [18]. For some new developments on the fractional Langevin equation, see, for example, [19-26].

The main objective of this paper is to develop the existence theory for a class of problems of the type (1), when the right-hand side is convex as well as nonconvex valued. We establish three existence results: the first result is obtained by means of the nonlinear alternative of Leray-Schauder type; the second one relies on the nonlinear alternative of Leray-Schauder type for single-valued maps together with a selection theorem due to Bressan and Colombo for lower semicontinuous multivalued maps with nonempty closed and decomposable values; and a fixed point theorem due to Covitz and Nadler for contraction multivalued maps is applied to get 
the third result. The methods used are well known; however their exposition in the framework of problem (1) is new.

The paper is organized as follows: in Section 2, we recall some preliminary facts that we need in the sequel, and Section 3 contains our main results.

\section{Preliminaries}

2.1. Fractional Calculus. Let us recall some basic definitions of fractional calculus [1-3].

Definition 1. For at least $n$-times differentiable function $g$ : $[0, \infty) \rightarrow \mathbb{R}$, the Caputo derivative of fractional order $q$ is defined as

$$
\begin{array}{r}
{ }^{c} D^{q} g(t)=\frac{1}{\Gamma(n-q)} \int_{0}^{t}(t-s)^{n-q-1} g^{(n)}(s) d s, \\
n-1<q<n, n=[q]+1,
\end{array}
$$

where $[q]$ denotes the integer part of the real number $q$.

Definition 2. The Riemann-Liouville fractional integral of order $q$ is defined as

$$
I^{q} g(t)=\frac{1}{\Gamma(q)} \int_{0}^{t} \frac{g(s)}{(t-s)^{1-q}} d s, \quad q>0,
$$

provided the integral exists.

Lemma 3. For $q>0$, the general solution of the fractional differential equation $D^{q} x(t)=0$ is given by

$$
x(t)=c_{0}+c_{1} t+\cdots+c_{n-1} t^{n-1},
$$

where $c_{i} \in \mathbb{R}, i=1,2, \ldots, n-1(n=[q]+1)$.

In view of Lemma 3, it follows that

$$
I^{q} D^{q} x(t)=x(t)+c_{0}+c_{1} t+\cdots+c_{n-1} t^{n-1},
$$

for some $c_{i} \in \mathbb{R}, i=1,2, \ldots, n-1(n=[q]+1)$.

In the following, $A C^{1}([0, T], \mathbb{R})$ will denote the space of functions $x:[0, T] \rightarrow \mathbb{R}$ that are absolutely continuous and whose first derivative is absolutely continuous.

Definition 4. A function $x \in A C^{1}([0, T], \mathbb{R})$ is called a solution of problem $(1)$ if there exists a function $v \in L^{1}([0, T], \mathbb{R})$ with $v(t) \in F(t, x(t))$, a.e. $[0, T]$ such that ${ }^{c} D^{p}\left({ }^{c} D^{q}+\right.$ $\lambda) x(t)=v(t)$, a.e. $[0, T]$, and $x(0)=\sum_{i=1}^{n} \beta_{i}\left(I^{\mu_{i}} x\right)(\zeta), x(1)=$ $\sum_{i=1}^{n} \alpha_{i}\left(I^{v_{i}} x\right)(\eta)$.

Lemma 5. Let $h \in C[0,1]$. Then the boundary value problem

$$
\begin{gathered}
{ }^{c} D^{p}\left({ }^{c} D^{q}+\lambda\right) x(t)=h(t), \quad 0<t<1, \\
x(0)=\sum_{i=1}^{n} \beta_{i}\left(I^{\mu_{i}} x\right)(\zeta), \\
x(1)=\sum_{i=1}^{n} \alpha_{i}\left(I^{\nu_{i}} x\right)(\eta), \quad 0<\zeta<\eta<1,
\end{gathered}
$$

has a unique solution

$$
\begin{aligned}
x(t)=\int_{0}^{t} \frac{(t-s)^{q-1}}{\Gamma(q)} & \\
& \times\left(\int_{0}^{s} \frac{(s-r)^{p-1}}{\Gamma(p)} h(r) d r-\lambda x(s)\right) d s \\
- & \frac{1}{\Delta}\left(\frac{t^{q} \Delta_{3}+\Delta_{1} \Gamma(q+1)}{\Gamma(q+1)}\right) \\
\times & {\left[\int_{0}^{1} \frac{(1-s)^{q-1}}{\Gamma(q)}\right.} \\
& \times\left(\int_{0}^{s} \frac{(s-r)^{p-1}}{\Gamma(p)} h(r) d r-\lambda x(s)\right) d s \\
& \quad-\sum_{i=1}^{n} \alpha_{i} \int_{0}^{\eta} \frac{(\eta-u)^{v_{i}-1}}{\Gamma\left(v_{i}\right)}
\end{aligned}
$$

$$
\begin{aligned}
& \times\left(\int_{0}^{u} \frac{(u-s)^{q-1}}{\Gamma(q)}\right. \\
& \times\left\{\int_{0}^{s} \frac{(s-r)^{p-1}}{\Gamma(p)} h(r) d r\right. \\
& \quad-\lambda x(s)\} d s) d u]
\end{aligned}
$$

$$
\begin{aligned}
& -\frac{1}{\Delta}\left(\frac{t^{q} \Delta_{2}+\Delta_{4} \Gamma(q+1)}{\Gamma(q+1)}\right) \\
& \times \sum_{i=1}^{n} \beta_{i} \int_{0}^{\zeta} \frac{(\zeta-u)^{\mu_{i}-1}}{\Gamma\left(\mu_{i}\right)} \\
& \times\left(\int_{0}^{u} \frac{(u-s)^{q-1}}{\Gamma(q)}\right. \\
& \left.\times\left\{\int_{0}^{s} \frac{(s-r)^{p-1}}{\Gamma(p)} h(r) d r-\lambda x(s)\right\} d s\right) d u,
\end{aligned}
$$

where

$$
\begin{aligned}
\Delta & =\Delta_{1} \Delta_{2}-\Delta_{3} \Delta_{4} \neq 0, \\
\Delta_{1} & =\sum_{i=1}^{n} \frac{\beta_{i} \zeta^{\mu_{i}+q}}{\Gamma\left(\mu_{i}+q+1\right)}, \\
\Delta_{2} & =\left(1-\sum_{i=1}^{n} \frac{\alpha_{i} \eta^{\nu_{i}}}{\Gamma\left(\nu_{i}+1\right)}\right), \\
\Delta_{3} & =\left(1-\sum_{i=1}^{n} \frac{\beta_{i} \zeta^{\mu_{i}}}{\Gamma\left(\mu_{i}+1\right)}\right), \\
\Delta_{4} & =\left(\sum_{i=1}^{n} \frac{\alpha_{i} \eta^{\nu_{i}+q}}{\Gamma\left(\nu_{i}+q+1\right)}-\frac{1}{\Gamma(q+1)}\right) .
\end{aligned}
$$


Proof. As argued in [23], the solution of ${ }^{c} D^{p}\left({ }^{c} D^{q}+\lambda\right) x(t)=$ $h(t)$ can be written as

$$
\begin{aligned}
x(t)= & \int_{0}^{t} \frac{(t-s)^{q-1}}{\Gamma(q)} \\
& \times\left(\int_{0}^{s} \frac{(s-r)^{p-1}}{\Gamma(p)} h(r) d r-\lambda x(s)\right) d s \\
& -\frac{c_{0}}{\Gamma(q+1)} t^{q}-c_{1} .
\end{aligned}
$$

Using the given conditions in (9) together with (8), we find that

$$
\begin{gathered}
\Delta_{3} c_{1}-\Delta_{1} c_{0}=-\mathscr{J}_{1}, \\
\Delta_{2} c_{1}-\Delta_{4} c_{0}=\mathscr{J}_{2},
\end{gathered}
$$

where

$$
\begin{gathered}
\mathscr{J}_{1}=\sum_{i=1}^{n} \beta_{i} \int_{0}^{\zeta} \frac{(\zeta-u)^{\mu_{i}-1}}{\Gamma\left(\mu_{i}\right)} \\
\quad \times\left(\int_{0}^{u} \frac{(u-s)^{q-1}}{\Gamma(q)}\right. \\
\left.\quad \times\left\{\int_{0}^{s} \frac{(s-r)^{p-1}}{\Gamma(p)} h(r) d r-\lambda x(s)\right\} d s\right) d u, \\
\mathscr{J}_{2}=\int_{0}^{1} \frac{(1-s)^{q-1}}{\Gamma(q)} \\
\quad \times\left(\int_{0}^{s} \frac{(s-r)^{p-1}}{\Gamma(p)} h(r) d r-\lambda x(s)\right) d s \\
-\sum_{i=1}^{n} \alpha_{i} \int_{0}^{\eta} \frac{(\eta-u)^{v_{i}-1}}{\Gamma\left(v_{i}\right)} \\
\times\left(\int_{0}^{u} \frac{(u-s)^{q-1}}{\Gamma(q)}\right. \\
\left.\times\left\{\int_{0}^{s} \frac{(s-r)^{p-1}}{\Gamma(p)} h(r) d r-\lambda x(s)\right\} d s\right) d u .
\end{gathered}
$$

Solving (10) for $c_{0}$ and $c_{1}$, we find that

$$
\begin{aligned}
& c_{0}=\frac{1}{\Delta}\left(\Delta_{2} \mathscr{J}_{1}+\Delta_{3} \mathscr{J}_{2}\right), \\
& c_{1}=\frac{1}{\Delta}\left(\Delta_{4} \mathscr{J}_{1}+\Delta_{1} \mathscr{J}_{2}\right) .
\end{aligned}
$$

Substituting these values in (9), we find the desired solution.

In order to simplify the computations in the main results, we present a technical lemma, concerning the bounds of the operators $\mathscr{J}_{1}$ and $\mathscr{J}_{2}$ defined in the proof of the above lemma.
Lemma 6. One has

$$
\begin{aligned}
\left|\mathscr{J}_{1}\right| \leq & \sum_{i=1}^{n}\left|\beta_{i}\right| \frac{\zeta^{\mu_{i}+p+q}\|h\|}{\Gamma\left(\mu_{i}+p+q+1\right)} \\
& +\sum_{i=1}^{n}\left|\beta_{i}\right| \frac{\zeta^{\mu_{i}+q}|\lambda|\|x\|}{\Gamma\left(\mu_{i}+q+1\right)}, \\
\left|\mathscr{J}_{2}\right| \leq & \frac{\|h\|}{\Gamma(p+q+1)}+\frac{|\lambda|\|x\|}{\Gamma(q+1)} \\
& +\sum_{i=1}^{n}\left|\alpha_{i}\right| \frac{\eta^{\nu_{i}+p+q}\|h\|}{\Gamma\left(\nu_{i}+p+q+1\right)} \\
& +\sum_{i=1}^{n}\left|\alpha_{i}\right| \frac{\eta^{\nu_{i}+q}|\lambda|\|x\|}{\Gamma\left(\nu_{i}+q+1\right)} .
\end{aligned}
$$

Proof. By using the following property of beta function

$$
\begin{aligned}
B(b+1, a) & =\int_{0}^{1}(1-s)^{a-1} s^{b} d s \\
& =\frac{\Gamma(a) \Gamma(b+1)}{\Gamma(a+b+1)},
\end{aligned}
$$

we have

$$
\begin{aligned}
\left|\mathscr{J}_{1}\right| \leq & \sum_{i=1}^{n}\left|\beta_{i}\right| \\
& \times \int_{0}^{\zeta} \frac{(\zeta-u)^{\mu_{i}-1}}{\Gamma\left(\mu_{i}\right)} \\
& \times\left(\int_{0}^{u} \frac{(u-s)^{q-1}}{\Gamma(q)}\right. \\
& \left.\times\left\{\int_{0}^{s} \frac{(s-r)^{p-1}}{\Gamma(p)}|h(r)| d r+|\lambda||x(s)|\right\} d s\right) d u \\
\leq & \sum_{i=1}^{n}\left|\beta_{i}\right| \int_{0}^{\zeta} \frac{(\zeta-u)^{\mu_{i}-1}}{\Gamma\left(\mu_{i}\right)}
\end{aligned}
$$$$
\times\left(\int_{0}^{u} \frac{(u-s)^{q-1}}{\Gamma(q)}\right.
$$$$
\left.\times\left\{\frac{s^{p}}{\Gamma(p+1)}\|h\|+|\lambda|\|x\|\right\} d s\right) d u
$$

$$
\begin{aligned}
& \leq \sum_{i=1}^{n}\left|\beta_{i}\right| \int_{0}^{\zeta} \frac{(\zeta-u)^{\mu_{i}-1}}{\Gamma\left(\mu_{i}\right)} \\
& \quad \times\left(\int_{0}^{u} \frac{(u-s)^{q-1} s^{p}\|h\|}{\Gamma(q) \Gamma(p+1)} d s\right. \\
& \left.\quad+\int_{0}^{u} \frac{(u-s)^{q-1}}{\Gamma(q)}|\lambda|\|x\| d s\right) d u
\end{aligned}
$$




$$
\begin{aligned}
& \leq \sum_{i=1}^{n}\left|\beta_{i}\right| \int_{0}^{\zeta} \frac{(\zeta-u)^{\mu_{i}-1}}{\Gamma\left(\mu_{i}\right)} \frac{u^{p+q}\|h\|}{\Gamma(q) \Gamma(p+1)} \\
& \quad \times B(p+1, q) d u \\
& \quad+\sum_{i=1}^{n}\left|\beta_{i}\right| \int_{0}^{\zeta} \frac{(\zeta-u)^{\mu_{i}-1}}{\Gamma\left(\mu_{i}\right)} \frac{u^{q}}{\Gamma(q+1)}|\lambda|\|x\| d u \\
& \leq \sum_{i=1}^{n}\left|\beta_{i}\right| \frac{\zeta^{\mu_{i}+p+q}\|h\|}{\Gamma\left(\mu_{i}\right) \Gamma(q) \Gamma(p+1)} \\
& \quad \times B(p+1, q) B\left(p+q+1, \mu_{i}\right) \\
& \quad+\sum_{i=1}^{n}\left|\beta_{i}\right| \frac{\zeta^{\mu_{i}+q}}{\Gamma\left(\mu_{i}\right) \Gamma(q+1)} B\left(q+1, \mu_{i}\right)|\lambda|\|x\| \\
& =\sum_{i=1}^{n}\left|\beta_{i}\right| \frac{\zeta^{\mu_{i}+p+q}\|h\|}{\Gamma\left(\mu_{i}+p+q+1\right)} \\
& \quad+\sum_{i=1}^{n}\left|\beta_{i}\right| \frac{\zeta^{\mu_{i}+q}|\lambda|\|x\|}{\Gamma\left(\mu_{i}+q+1\right)} .
\end{aligned}
$$

By a similar way, we have

$$
\begin{aligned}
\left|\mathscr{J}_{2}\right| \leq & \frac{|v(u)|}{\Gamma(p+q+1)}+\frac{|\lambda||x(u)|}{\Gamma(q+1)} \\
& +\sum_{i=1}^{n}\left|\alpha_{i}\right| \frac{\eta^{\nu_{i}+p+q}|v(u)|}{\Gamma\left(\nu_{i}+p+q+1\right)} \\
& +\sum_{i=1}^{n}\left|\alpha_{i}\right| \frac{\eta^{\nu_{i}+q}|\lambda||x(u)|}{\Gamma\left(\nu_{i}+q+1\right)},
\end{aligned}
$$

which completes the proof.

In the following, for convenience, we put

$$
\begin{gathered}
A_{1}=\frac{\left|\Delta_{3}\right|+\left|\Delta_{1}\right| \Gamma(q+1)}{|\Delta| \Gamma(q+1)}, \\
A_{2}=\frac{\left|\Delta_{2}\right|+\left|\Delta_{4}\right| \Gamma(q+1)}{|\Delta| \Gamma(q+1)}, \\
\Omega=\frac{1+A_{1}}{\Gamma(p+q+1)} \\
+A_{1} \sum_{i=1}^{n}\left|\alpha_{i}\right| \frac{\eta^{\nu_{i}+p+q}}{\Gamma\left(\nu_{i}+p+q+1\right)} \\
+A_{2} \sum_{i=1}^{n}\left|\beta_{i}\right| \frac{\zeta^{\mu_{i}+p+q}}{\Gamma\left(\mu_{i}+p+q+1\right)},
\end{gathered}
$$

$$
\begin{aligned}
\Psi= & \frac{|\lambda|\left(1+A_{1}\right)}{\Gamma(q+1)} \\
& +|\lambda| A_{1} \sum_{i=1}^{n}\left|\alpha_{i}\right| \frac{\eta^{\nu_{i}+q}}{\Gamma\left(\nu_{i}+q+1\right)} \\
& +A_{2} \sum_{i=1}^{n}\left|\beta_{i}\right| \frac{\zeta^{\mu_{i}+q}}{\Gamma\left(\mu_{i}+q+1\right)} .
\end{aligned}
$$

2.2. Background Material for Multivalued Analysis. Now we recall some basic definitions on multivalued maps [27-29].

Let $C([0,1])$ denote a Banach space of continuous functions from $[0,1]$ into $\mathbb{R}$ with the norm $\|x\|=\sup _{t \in[0,1]}|x(t)|$. Let $L^{1}([0,1], \mathbb{R})$ be the Banach space of measurable functions $x:[0,1] \rightarrow \mathbb{R}$ which are Lebesgue integrable and normed by $\|x\|_{L^{1}}=\int_{0}^{1}|x(t)| d t$.

For a normed space $(X,\|\cdot\|)$, let

$$
\begin{gathered}
\mathscr{P}_{\mathrm{cl}}(X)=\{Y \in \mathscr{P}(X): Y \text { is closed }\}, \\
\mathscr{P}_{b}(X)=\{Y \in \mathscr{P}(X): Y \text { is bounded }\}, \\
\mathscr{P}_{c p}(X)=\{Y \in \mathscr{P}(X): Y \text { is compact }\}, \\
\mathscr{P}_{c p, c}(X)=\{Y \in \mathscr{P}(X): Y \text { is compact and convex }\} .
\end{gathered}
$$

A multivalued map $G: X \rightarrow \mathscr{P}(X)$

(i) is convex (closed) valued if $G(x)$ is convex (closed) for all $x \in X$;

(ii) is bounded on bounded sets if $G(\mathbb{B})=\cup_{x \in \mathbb{B}} G(x)$ is bounded in $X$ for all $\mathbb{B} \in \mathscr{P}_{b}(X)$ (i.e., $\sup _{x \in \mathbb{B}}\{\sup \{|y|$ : $y \in G(x)\}\}<\infty)$

(iii) is called upper semicontinuous (u.s.c.) on $X$ if, for each $x_{0} \in X$, the set $G\left(x_{0}\right)$ is a nonempty closed subset of $X$ and if, for each open set $N$ of $X$ containing $G\left(x_{0}\right)$, there exists an open neighborhood $\mathcal{N}_{0}$ of $x_{0}$ such that $G\left(\mathcal{N}_{0}\right) \subseteq N$;

(iv) $G$ is lower semicontinuous (l.s.c.) if the set $\{y \in X$ : $G(y) \cap B \neq \emptyset\}$ is open for any open set $B$ in $E$;

(v) is said to be completely continuous if $G(\mathbb{B})$ is relatively compact for every $\mathbb{B} \in \mathscr{P}_{b}(X)$;

(vi) is said to be measurable if, for every $y \in \mathbb{R}$, the function

$$
t \longmapsto d(y, G(t))=\inf \{|y-z|: z \in G(t)\}
$$

is measurable;

(vii) has a fixed point if there is $x \in X$ such that $x \in G(x)$. The fixed point set of the multivalued operator $G$ will be denoted by FixG.

Definition 7. A multivalued map $F:[0,1] \times \mathbb{R} \rightarrow \mathscr{P}(\mathbb{R})$ is said to be Carathéodory if

(i) $t \mapsto F(t, x)$ is measurable for each $x \in \mathbb{R}$; 
(ii) $x \mapsto F(t, x)$ is upper semicontinuous for almost all $t \in[0,1]$

Further a Carathéodory function $F$ is called $L^{1}$-Carathéodory if

(iii) for each $\alpha>0$, there exists $\varphi_{\alpha} \in L^{1}\left([0,1], \mathbb{R}^{+}\right)$such that

$$
\|F(t, x)\|=\sup \{|v|: v \in F(t, x)\} \leq \varphi_{\alpha}(t)
$$

for all $\|x\|_{\infty} \leq \alpha$ and for a. e. $t \in[0,1]$. by

For each $x \in C([0,1], \mathbb{R})$, define the set of selections of $F$

$$
\begin{aligned}
& S_{F, x} \\
& :=\left\{v \in L^{1}([0,1], \mathbb{R}): v(t) \in F(t, x(t)) \text { for a.e. } t \in[0,1]\right\} .
\end{aligned}
$$

We define the graph of $G$ to be the set $G r(G)=\{(x, y) \in$ $X \times Y, y \in G(x)\}$ and recall two useful results regarding closed graphs and upper semicontinuity.

Lemma 8 (see [27, Proposition 1.2]). If $G: X \rightarrow \mathscr{P}_{\mathrm{cl}}(Y)$ is u.s.c., then $\operatorname{Gr}(G)$ is a closed subset of $X \times Y$; that is, for every sequence $\left\{x_{n}\right\}_{n \in \mathbb{N}} \subset X$ and $\left\{y_{n}\right\}_{n \in \mathbb{N}} \subset Y$, if, when $n \rightarrow \infty, x_{n} \rightarrow x_{*}, y_{n} \rightarrow y_{*}$, and $y_{n} \in G\left(x_{n}\right)$, then $y_{*} \in G\left(x_{*}\right)$. Conversely, if $G$ is completely continuous and has a closed graph, then it is upper semicontinuous.

Lemma 9 (see [30]). Let $X$ be a Banach space. Let $F:[0, T] \times$ $\mathbb{R} \rightarrow \mathscr{P}_{c p, c}(X)$ be an $L^{1}$-Carathéodory multivalued map, and let $\Theta$ be a linear continuous mapping from $L^{1}([0,1], X)$ to $C([0,1], X)$. Then the operator

$$
\begin{gathered}
\Theta \circ S_{F}: C([0,1], X) \longrightarrow \mathscr{P}_{c p, c}(C([0,1], X)), \\
x \longmapsto\left(\Theta \circ S_{F}\right)(x)=\Theta\left(S_{F, x, y}\right)
\end{gathered}
$$

is a closed graph operator in $C([0,1], X) \times C([0,1], X)$.

We recall the well-known nonlinear alternative of LeraySchauder for multivalued maps.

Lemma 10 (nonlinear alternative for Kakutani maps [31]). Let $E$ be a Banach space, $C$ a closed convex subset of $E, U$ an open subset of $C$, and $0 \in U$. Suppose that $F: \bar{U} \rightarrow \mathscr{P}_{c, c v}(C)$ is an upper semicontinuous compact map; here $\mathscr{P}_{c, c v}(C)$ denotes the family of nonempty, compact convex subsets of $C$. Then either

(i) F has a fixed point in $\bar{U}$, or

(ii) there is $a u \in \partial U$ and $\lambda \in(0,1)$ with $u \in \lambda F(u)$.

Definition 11. Let $A$ be a subset of $[0,1] \times \mathbb{R} . A$ is $\mathscr{L} \otimes \mathscr{B}$ measurable if $A$ belongs to the $\sigma$-algebra generated by all sets of the form $\mathscr{J} \times \mathscr{D}$, where $\mathscr{J}$ is Lebesgue measurable in $[0,1]$ and $\mathscr{D}$ is Borel measurable in $\mathbb{R}$.
Definition 12. A subset $\mathscr{A}$ of $L^{1}([0,1], \mathbb{R})$ is decomposable if, for all $u, v \in \mathscr{A}$, and measurable $\mathscr{J} \subset[0,1]=J$, the function $u \chi_{\mathcal{F}}+v \chi_{J-\mathcal{F}} \in \mathscr{A}$, where $\chi_{\mathcal{g}}$ stands for the characteristic function of $\mathscr{J}$.

Lemma 13 (see [32]). Let $Y$ be a separable metric space, and let $N: Y \rightarrow \mathscr{P}\left(L^{1}([0,1], \mathbb{R})\right)$ be a lower semicontinuous (l.s.c.) multivalued operator with nonempty closed and decomposable values. Then $N$ has a continuous selection; that is, there exists a continuous function (single-valued) $g: Y \rightarrow L^{1}([0,1], \mathbb{R}$ ) such that $g(x) \in N(x)$ for every $x \in Y$.

Let $(X, d)$ be a metric space induced from the normed space $(X ;\|\cdot\|)$. Consider $H_{d}: \mathscr{P}(X) \times \mathscr{P}(X) \rightarrow \mathbb{R} \cup\{\infty\}$ given by

$$
H_{d}(A, B)=\max \left\{\sup _{a \in A} d(a, B), \sup _{b \in B} d(A, b)\right\},
$$

where $d(A, b)=\inf _{a \in A} d(a ; b)$ and $d(a, B)=\inf _{b \in B} d(a ; b)$. Then $\left(\mathscr{P}_{b, \mathrm{cl}}(X), H_{d}\right)$ is a metric space (see [33]).

Definition 14. A multivalued operator $N: X \rightarrow \mathscr{P}_{\mathrm{cl}}(X)$ is called

(a) $\gamma$-Lipschitz if and only if there exists $\gamma>0$ such that

$H_{d}(N(x), N(y)) \leq \gamma d(x, y) \quad$ for each $x, y \in X ;$

(b) a contraction if and only if it is $\gamma$-Lipschitz with $\gamma<1$.

Lemma 15 (see [34]). Let $(X, d)$ be a complete metric space. If $N: X \rightarrow \mathscr{P}_{\mathrm{cl}}(X)$ is a contraction, then Fix $N \neq \emptyset$.

\section{Main Results}

3.1. The Carathéodory Case. In this section, we are concerned with the existence of solutions for the problem (1) when the right-hand side has convex as well as nonconvex values. Initially, we assume that $F$ is a compact and convex valued multivalued map.

Theorem 16. Suppose that

$\left(H_{1}\right)$ the map $F:[0,1] \times \mathbb{R} \rightarrow \mathscr{P}(\mathbb{R})$ is Carathéodory and has nonempty compact and convex values;

$\left(\mathrm{H}_{2}\right)$ there exist a continuous nondecreasing function $\psi$ : $[0, \infty) \rightarrow(0, \infty)$ and function $p \in L^{1}\left([0,1], \mathbb{R}^{+}\right)$ such that

$\|F(t, x)\|_{\mathscr{P}}:=\sup \{|v|: v \in F(t, x)\} \leq p(t) \psi(\|x\|)$

for each $(t, u) \in[0,1] \times \mathbb{R}$;

$\left(\mathrm{H}_{3}\right)$ there exists a number $M>0$ such that

$$
\frac{M(1-\Psi)}{\psi(M)\|p\|_{L^{1}} \Omega}>1,
$$

with $\Psi<1$, where $\Omega, \Psi$ are defined in (17).

Then BVP (1) has at least one solution.

Proof. Let us introduce the operator $N: C([0,1], \mathbb{R}) \rightarrow$ $\mathscr{P}(C([0,1], \mathbb{R}))$ as 


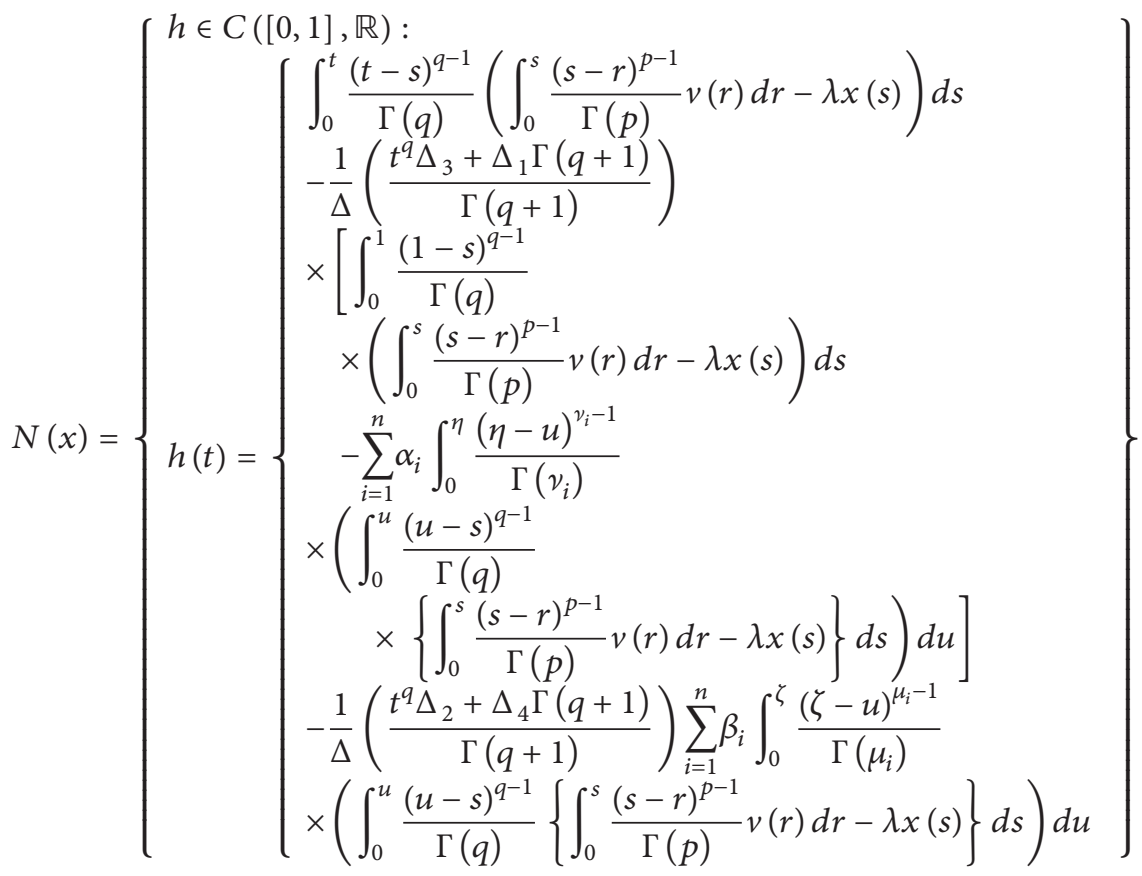

for $v \in S_{F, x}$. We will show that the operator $N$ satisfies the assumptions of the nonlinear alternative of Leray-Schauder type. The proof consists of several steps. As a first step, we show that $N(x)$ is convex for each $x \in C([0,1], \mathbb{R})$. For that, let $h_{1}, h_{2} \in N(x)$. Then there exist $v_{1}, v_{2} \in S_{F, x}$ such that, for each $t \in[0,1]$, we have

$$
\begin{aligned}
h_{i}(t) & \int_{0}^{t} \frac{(t-s)^{q-1}}{\Gamma(q)} \\
& \times\left(\int_{0}^{s} \frac{(s-r)^{p-1}}{\Gamma(p)} v_{i}(r) d r-\lambda x(s)\right) d s \\
- & \frac{1}{\Delta}\left(\frac{t^{q} \Delta_{3}+\Delta_{1} \Gamma(q+1)}{\Gamma(q+1)}\right) \\
\times & {\left[\int_{0}^{1} \frac{(1-s)^{q-1}}{\Gamma(q)}\right.} \\
& \times\left(\int_{0}^{s} \frac{(s-r)^{p-1}}{\Gamma(p)} v_{i}(r) d r-\lambda x(s)\right) d s \\
& -\sum_{i=1}^{n} \alpha_{i} \int_{0}^{\eta} \frac{(\eta-u)^{v_{i}-1}}{\Gamma\left(v_{i}\right)} \\
& \times\left(\int_{0}^{u} \frac{(u-s)^{q-1}}{\Gamma(q)}\right. \\
& \left.\left.\times\left\{\int_{0}^{s} \frac{(s-r)^{p-1}}{\Gamma(p)} v_{i}(r) d r-\lambda x(s)\right\} d s\right) d u\right]
\end{aligned}
$$

$$
\begin{aligned}
& -\frac{1}{\Delta}\left(\frac{t^{q} \Delta_{2}+\Delta_{4} \Gamma(q+1)}{\Gamma(q+1)}\right) \\
& \times \sum_{i=1}^{n} \beta_{i} \\
& \quad \times \int_{0}^{\zeta} \frac{(\zeta-u)^{\mu_{i}-1}}{\Gamma\left(\mu_{i}\right)} \\
& \quad \times\left(\int_{0}^{u} \frac{(u-s)^{q-1}}{\Gamma(q)}\right. \\
& \left.\quad \times\left\{\int_{0}^{s} \frac{(s-r)^{p-1}}{\Gamma(p)} v_{i}(r) d r-\lambda x(s)\right\} d s\right) d u,
\end{aligned}
$$

for $i=1,2$. Let $0 \leq \omega \leq 1$. Then, for each $t \in[0,1]$ and putting $z(r)=\omega v_{1}(r)+(1-\omega) v_{2}(r)$, we have

$$
\begin{aligned}
& {\left[\omega h_{1}+(1-\omega) h_{2}\right](t)} \\
& =\int_{0}^{t} \frac{(t-s)^{q-1}}{\Gamma(q)}\left(\int_{0}^{s} \frac{(s-r)^{p-1}}{\Gamma(p)} z(r) d r-\lambda x(s)\right) d s \\
& -\frac{1}{\Delta}\left(\frac{t^{q} \Delta_{3}+\Delta_{1} \Gamma(q+1)}{\Gamma(q+1)}\right) \\
& \times\left[\int_{0}^{1} \frac{(1-s)^{q-1}}{\Gamma(q)}\left(\int_{0}^{s} \frac{(s-r)^{p-1}}{\Gamma(p)} z(r) d r-\lambda x(s)\right) d s\right. \\
& \quad-\sum_{i=1}^{n} \alpha_{i} \int_{0}^{\eta} \frac{(\eta-u)^{v_{i}-1}}{\Gamma\left(v_{i}\right)}
\end{aligned}
$$




$$
\begin{aligned}
& \times\left(\int_{0}^{u} \frac{(u-s)^{q-1}}{\Gamma(q)}\right. \\
& \left.\left.\times\left\{\int_{0}^{s} \frac{(s-r)^{p-1}}{\Gamma(p)} z(r) d r-\lambda x(s)\right\} d s\right) d u\right] \\
& -\frac{1}{\Delta}\left(\frac{t^{q} \Delta_{2}+\Delta_{4} \Gamma(q+1)}{\Gamma(q+1)}\right) \\
& \times \sum_{i=1}^{n} \beta_{i} \int_{0}^{\zeta} \frac{(\zeta-u)^{\mu_{i}-1}}{\Gamma\left(\mu_{i}\right)} \\
& \times\left(\int_{0}^{u} \frac{(u-s)^{q-1}}{\Gamma(q)}\right. \\
& \left.\times\left\{\int_{0}^{s} \frac{(s-r)^{p-1}}{\Gamma(p)} z(r) d r-\lambda x(s)\right\} d s\right) d u .
\end{aligned}
$$

Since $S_{F, x}$ is convex ( $F$ has convex values), therefore it follows that $\omega h_{1}+(1-\omega) h_{2} \in N(x)$.

Next, we show that $N(x)$ maps bounded sets into bounded sets in $C([0,1], \mathbb{R})$. For a positive number $r$, let $B_{\rho}=\{x \in$ $C([0,1], \mathbb{R}):\|x\| \leq \rho\}$ be a bounded set in $C([0,1], \mathbb{R})$. Then, for each $h \in N(x), x \in B_{\rho}$, there exists $v \in S_{F, x}$ such that

$h(t)$

$$
\begin{aligned}
& =\int_{0}^{t} \frac{(t-s)^{q-1}}{\Gamma(q)} \\
& \times\left(\int_{0}^{s} \frac{(s-r)^{p-1}}{\Gamma(p)} v(r) d r-\lambda x(s)\right) d s \\
& -\frac{1}{\Delta}\left(\frac{t^{q} \Delta_{3}+\Delta_{1} \Gamma(q+1)}{\Gamma(q+1)}\right) \\
& \times\left[\int_{0}^{1} \frac{(1-s)^{q-1}}{\Gamma(q)}\right. \\
& \times\left(\int_{0}^{s} \frac{(s-r)^{p-1}}{\Gamma(p)} v(r) d r-\lambda x(s)\right) d s \\
& -\sum_{i=1}^{n} \alpha_{i} \int_{0}^{\eta} \frac{(\eta-u)^{v_{i}-1}}{\Gamma\left(\nu_{i}\right)} \\
& \times\left(\int_{0}^{u} \frac{(u-s)^{q-1}}{\Gamma(q)}\right. \\
& \left.\left.\times\left\{\int_{0}^{s} \frac{(s-r)^{p-1}}{\Gamma(p)} v(r) d r-\lambda x(s)\right\} d s\right) d u\right] \\
& -\frac{1}{\Delta}\left(\frac{t^{q} \Delta_{2}+\Delta_{4} \Gamma(q+1)}{\Gamma(q+1)}\right) \\
& \times \sum_{i=1}^{n} \beta_{i} \int_{0}^{\zeta} \frac{(\zeta-u)^{\mu_{i}-1}}{\Gamma\left(\mu_{i}\right)}
\end{aligned}
$$

$$
\begin{aligned}
\times & \int_{0}^{u} \frac{(u-s)^{q-1}}{\Gamma(q)} \\
& \left.\times\left\{\int_{0}^{s} \frac{(s-r)^{p-1}}{\Gamma(p)} v(r) d r-\lambda x(s)\right\} d s\right) d u \\
= & \int_{0}^{t} \frac{(t-s)^{q-1}}{\Gamma(q)} \\
& \times\left(\int_{0}^{s} \frac{(s-r)^{p-1}}{\Gamma(p)} v(\mathrm{r}) d r-\lambda x(s)\right) d s \\
- & \frac{1}{\Delta}\left(\frac{t^{q} \Delta_{3}+\Delta_{1} \Gamma(q+1)}{\Gamma(q+1)}\right) \mathscr{J}_{2} \\
- & \frac{1}{\Delta}\left(\frac{t^{q} \Delta_{2}+\Delta_{4} \Gamma(q+1)}{\Gamma(q+1)}\right) \mathscr{J}_{1},
\end{aligned}
$$

$|h(t)|$

$$
\begin{aligned}
& \leq \int_{0}^{t} \frac{(t-s)^{q-1}}{\Gamma(q)} \\
& \times\left(\int_{0}^{s} \frac{(s-r)^{p-1}}{\Gamma(p)}|v(r)| d r+|\lambda||x(s)|\right) d s \\
& +A_{1}\left|\mathscr{J}_{2}\right|+A_{2}\left|\mathscr{J}_{1}\right| \\
& \leq \frac{\psi(\|x\|)\|p\|_{L^{1}}}{\Gamma(p+q+1)}+\frac{|\lambda|\|x\|}{\Gamma(q+1)} \\
& +A_{1}\left(\frac{\psi(\|x\|)\|p\|_{L^{1}}}{\Gamma(p+q+1)}+\frac{|\lambda|\|x\|}{\Gamma(q+1)}\right. \\
& +\sum_{i=1}^{n}\left|\alpha_{i}\right|\left[\frac{\eta^{\nu_{i}+p+q} \psi(\|x\|)\|p\|_{L^{1}}}{\Gamma\left(\nu_{i}+p+q+1\right)}\right. \\
& \left.\left.+\frac{\eta^{\nu_{i}+q}|\lambda|\|x\|}{\Gamma\left(\nu_{i}+q+1\right)}\right]\right) \\
& +A_{2} \sum_{i=1}^{n}\left|\beta_{i}\right|\left(\frac{\zeta^{\mu_{i}+p+q} \psi(\|x\|)\|p\|_{L^{1}}}{\Gamma\left(\mu_{i}+p+q+1\right)}\right. \\
& \left.+\frac{\zeta^{\mu_{i}+q}|\lambda|\|x\|}{\Gamma\left(\mu_{i}+q+1\right)}\right) \\
& =\psi(\|x\|)\|p\|_{L^{1}} \\
& \times\left\{\frac{1+A_{1}}{\Gamma(p+q+1)}\right. \\
& +A_{1} \sum_{i=1}^{n}\left|\alpha_{i}\right| \frac{\eta^{\nu_{i}+p+q}}{\Gamma\left(\nu_{i}+p+q+1\right)} \\
& \left.+A_{2} \sum_{i=1}^{n}\left|\beta_{i}\right| \frac{\zeta^{\mu_{i}+p+q}}{\Gamma\left(\mu_{i}+p+q+1\right)}\right\}
\end{aligned}
$$




$$
\begin{aligned}
+\|x\|\left\{\frac{|\lambda|\left(1+A_{1}\right)}{\Gamma(q+1)}\right. \\
+|\lambda| A_{1} \sum_{i=1}^{n}\left|\alpha_{i}\right| \frac{\eta^{\nu_{i}+q}}{\Gamma\left(\nu_{i}+q+1\right)} \\
\left.+A_{2} \sum_{i=1}^{n}\left|\beta_{i}\right| \frac{\zeta^{\mu_{i}+q}}{\Gamma\left(\mu_{i}+q+1\right)}\right\}
\end{aligned}
$$$$
=\Omega \psi(\|x\|)\|p\|_{L^{1}}+\Psi\|x\|
$$

Then

$$
\|h\| \leq \Omega \psi(\rho)\|p\|_{L^{1}}+\Psi \rho
$$

Now we show that Nmaps bounded sets into equicontinuous sets of $C([0,1], \mathbb{R})$. Let $t^{\prime}, t^{\prime \prime} \in[0,1]$ with $t^{\prime}<t^{\prime \prime}$ and $x \in B_{\rho}$, where $B_{\rho}$, as above, is a bounded set of $C([0,1], \mathbb{R})$. For each $h \in N(x)$, we obtain

$$
\begin{aligned}
& \left|h\left(t^{\prime \prime}\right)-h\left(t^{\prime}\right)\right| \\
& \leq \mid \int_{0}^{t^{\prime \prime}} \frac{\left(t^{\prime \prime}-s\right)^{q-1}}{\Gamma(q)} \\
& \times\left(\int_{0}^{s} \frac{(s-r)^{p-1}}{\Gamma(p)}|v(r)| d r+|\lambda||x(s)|\right) d s \\
& -\int_{0}^{t^{\prime}} \frac{\left(t^{\prime}-s\right)^{q-1}}{\Gamma(q)} \\
& \times\left(\int_{0}^{s} \frac{(s-r)^{p-1}}{\Gamma(p)}|v(r)| d r+|\lambda||x(s)|\right) d s \mid \\
& +\frac{\left|\Delta_{3}\right|}{|\Delta| \Gamma(q+1)}\left|\left(t^{\prime \prime}\right)^{q}-\left(t^{\prime}\right)^{q}\right|\left|\mathscr{J}_{2}\right| \\
& +\frac{\left|\Delta_{2}\right|}{|\Delta| \Gamma(q+1)}\left|\left(t^{\prime \prime}\right)^{q}-\left(t^{\prime}\right)^{q}\right|\left|\mathscr{J}_{1}\right| \\
& \leq \mid \int_{0}^{t^{\prime}} \frac{\left[\left(t^{\prime \prime}-s\right)^{q-1}-\left(t^{\prime}-s\right)^{q-1}\right]}{\Gamma(q)} \\
& \times\left(\int_{0}^{s} \frac{(s-r)^{p-1}}{\Gamma(p)}|v(r)| d r+|\lambda||x(s)|\right) d s \\
& +\int_{t^{\prime}}^{t^{\prime \prime}} \frac{\left(t^{\prime \prime}-s\right)^{q-1}}{\Gamma(q)} \\
& \times\left(\int_{0}^{s} \frac{(s-r)^{p-1}}{\Gamma(p)}|v(r)| d r+|\lambda||x(s)|\right) d s \mid
\end{aligned}
$$

$$
\begin{aligned}
& +\frac{\left|\Delta_{3}\right|}{|\Delta| \Gamma(q+1)}\left|\left(t^{\prime \prime}\right)^{q}-\left(t^{\prime}\right)^{q}\right|\left|\mathscr{J}_{2}\right| \\
& +\frac{\left|\Delta_{2}\right|}{|\Delta| \Gamma(q+1)}\left|\left(t^{\prime \prime}\right)^{q}-\left(t^{\prime}\right)^{q}\right|\left|\mathscr{J}_{1}\right| \\
& \leq \mid \int_{0}^{t^{\prime}} \frac{\left[\left(t^{\prime \prime}-s\right)^{q-1}-\left(t^{\prime}-s\right)^{q-1}\right]}{\Gamma(q)} \\
& \times\left(\|p\|_{L^{1}} \psi(\rho) \int_{0}^{s} \frac{(s-r)^{p-1}}{\Gamma(p)} d r+|\lambda| \rho\right) d s \\
& +\int_{t^{\prime}}^{t^{\prime \prime}} \frac{\left(t^{\prime \prime}-s\right)^{q-1}}{\Gamma(q)} \\
& \times\left(\|p\|_{L^{1}} \psi(\rho) \int_{0}^{s} \frac{(s-r)^{p-1}}{\Gamma(p)} d r+|\lambda| \rho\right) d s \\
& +\frac{\left|\Delta_{3}\right|}{|\Delta| \Gamma(q+1)}\left|\left(t^{\prime \prime}\right)^{q}-\left(t^{\prime}\right)^{q}\right|\left|\mathscr{J}_{2}\right| \\
& +\frac{\left|\Delta_{2}\right|}{|\Delta| \Gamma(q+1)}\left|\left(t^{\prime \prime}\right)^{q}-\left(t^{\prime}\right)^{q}\right|\left|\mathscr{J}_{1}\right| \text {. }
\end{aligned}
$$

Obviously the right-hand side of the above inequality tends to zero independently of $x \in B_{\rho}$ as $t^{\prime \prime}-t^{\prime} \rightarrow$ 0 . As $N$ satisfies the above three assumptions, therefore it follows by Ascoli-Arzelá theorem that $N: C([0,1], \mathbb{R}) \rightarrow$ $\mathscr{P}(C([0,1], \mathbb{R}))$ is completely continuous.

In our next step, we show that $N$ has a closed graph. Let $x_{n} \rightarrow x_{*}, h_{n} \in N\left(x_{n}\right)$, and $h_{n} \rightarrow h_{*}$. Then we need to show that $h_{*} \in N\left(x_{*}\right)$. Associated with $h_{n} \in N\left(x_{n}\right)$, there exists $v_{n} \in S_{F, x_{n}}$ such that, for each $t \in[0,1]$,

$$
\begin{aligned}
h_{n}(t) & \int_{0}^{t} \frac{(t-s)^{q-1}}{\Gamma(q)} \\
& \times\left(\int_{0}^{s} \frac{(s-r)^{p-1}}{\Gamma(p)} v_{n}(r) d r-\lambda x(s)\right) d s \\
- & \frac{1}{\Delta}\left(\frac{t^{q} \Delta_{3}+\Delta_{1} \Gamma(q+1)}{\Gamma(q+1)}\right) \\
\times & {\left[\int_{0}^{1} \frac{(1-s)^{q-1}}{\Gamma(q)}\right.} \\
& \times\left(\int_{0}^{s} \frac{(s-r)^{p-1}}{\Gamma(p)} v_{n}(r) d r-\lambda x(s)\right) d s \\
& \quad-\sum_{\mathrm{i}=1}^{n} \alpha_{i} \int_{0}^{\eta} \frac{(\eta-u)^{v_{i}-1}}{\Gamma\left(v_{i}\right)}
\end{aligned}
$$




$$
\begin{aligned}
& \times\left(\int_{0}^{u} \frac{(u-s)^{q-1}}{\Gamma(q)}\right. \\
&\left.\left.\times\left\{\int_{0}^{s} \frac{(s-r)^{p-1}}{\Gamma(p)} v_{n}(r) d r-\lambda x(s)\right\} d s\right) d u\right] \\
&- \frac{1}{\Delta}\left(\frac{t^{q} \Delta_{2}+\Delta_{4} \Gamma(q+1)}{\Gamma(q+1)}\right) \\
& \times \sum_{i=1}^{n} \beta_{i} \int_{0}^{\zeta} \frac{(\zeta-u)^{\mu_{i}-1}}{\Gamma\left(\mu_{i}\right)} \times\left(\int_{0}^{u} \frac{(u-s)^{q-1}}{\Gamma(q)}\right. \\
&\left.\times\left\{\int_{0}^{s} \frac{(s-r)^{p-1}}{\Gamma(p)} v_{n}(r) d r-\lambda x(s)\right\} d s\right) d u .
\end{aligned}
$$

Thus we have to show that there exists $v_{*} \in S_{F, x_{*}}$ such that, for each $t \in[0,1]$,

$$
\begin{aligned}
& h_{*}(t) \\
& =\int_{0}^{t} \frac{(t-s)^{q-1}}{\Gamma(q)} \\
& \times\left(\int_{0}^{s} \frac{(s-r)^{p-1}}{\Gamma(p)} v_{*}(r) d r-\lambda x(s)\right) d s \\
& -\frac{1}{\Delta}\left(\frac{t^{q} \Delta_{3}+\Delta_{1} \Gamma(q+1)}{\Gamma(q+1)}\right) \\
& \times\left[\int_{0}^{1} \frac{(1-s)^{q-1}}{\Gamma(q)}\right. \\
& \times\left(\int_{0}^{s} \frac{(s-r)^{p-1}}{\Gamma(p)} v_{*}(r) d r-\lambda x(s)\right) d s \\
& -\sum_{i=1}^{n} \alpha_{i} \int_{0}^{\eta} \frac{(\eta-u)^{v_{i}-1}}{\Gamma\left(v_{i}\right)} \\
& \times\left(\int_{0}^{u} \frac{(u-s)^{q-1}}{\Gamma(q)}\right. \\
& \left.\left.\times\left\{\int_{0}^{s} \frac{(s-r)^{p-1}}{\Gamma(p)} v_{*}(r) d r-\lambda x(s)\right\} d s\right) d u\right] \\
& -\frac{1}{\Delta}\left(\frac{t^{q} \Delta_{2}+\Delta_{4} \Gamma(q+1)}{\Gamma(q+1)}\right) \\
& \times \sum_{i=1}^{n} \beta_{i} \int_{0}^{\zeta} \frac{(\zeta-u)^{\mu_{i}-1}}{\Gamma\left(\mu_{i}\right)} \\
& \times\left(\int_{0}^{u} \frac{(u-s)^{q-1}}{\Gamma(q)}\right. \\
& \left.\times\left\{\int_{0}^{s} \frac{(s-r)^{p-1}}{\Gamma(p)} v_{*}(r) d r-\lambda x(s)\right\} d s\right) d u .
\end{aligned}
$$

Let us consider the continuous linear operator $\Theta$ : $L^{1}([0,1], \mathbb{R}) \rightarrow C([0,1], \mathbb{R})$ so that

$$
\begin{aligned}
& v \longmapsto \Theta(v) \\
& =\int_{0}^{t} \frac{(t-s)^{q-1}}{\Gamma(q)} \\
& \times\left(\int_{0}^{s} \frac{(s-r)^{p-1}}{\Gamma(p)} v(r) d r-\lambda x(s)\right) d s \\
& -\frac{1}{\Delta}\left(\frac{t^{q} \Delta_{3}+\Delta_{1} \Gamma(q+1)}{\Gamma(q+1)}\right) \\
& \times\left[\int_{0}^{1} \frac{(1-s)^{q-1}}{\Gamma(q)}\right. \\
& \times\left(\int_{0}^{s} \frac{(s-r)^{p-1}}{\Gamma(p)} v(r) d r-\lambda x(s)\right) d s \\
& -\sum_{i=1}^{n} \alpha_{i} \\
& \times \int_{0}^{\eta} \frac{(\eta-u)^{v_{i}-1}}{\Gamma\left(v_{i}\right)} \\
& \times\left(\int_{0}^{u} \frac{(u-s)^{q-1}}{\Gamma(q)}\right. \\
& \left.\left.\times\left\{\int_{0}^{s} \frac{(s-r)^{p-1}}{\Gamma(p)} v(r) d r-\lambda x(s)\right\} d s\right) d u\right] \\
& -\frac{1}{\Delta}\left(\frac{t^{q} \Delta_{2}+\Delta_{4} \Gamma(q+1)}{\Gamma(q+1)}\right) \\
& \times \sum_{i=1}^{n} \beta_{i} \\
& \times \int_{0}^{\zeta} \frac{(\zeta-u)^{\mu_{i}-1}}{\Gamma\left(\mu_{i}\right)} \\
& \times\left(\int_{0}^{u} \frac{(u-s)^{q-1}}{\Gamma(q)}\right. \\
& \left.\times\left\{\int_{0}^{s} \frac{(s-r)^{p-1}}{\Gamma(p)} v(r) d r-\lambda x(s)\right\} d s\right) d u .
\end{aligned}
$$

Observe that

$\left\|h_{n}(t)-h_{*}(t)\right\|$

$=\| \int_{0}^{t} \frac{(t-s)^{q-1}}{\Gamma(q)}$

$$
\begin{aligned}
& \times\left(\int_{0}^{s} \frac{(s-r)^{p-1}}{\Gamma(p)}\left(v_{n}(r)-v_{*}(s)\right) d r\right) d s \\
& -\frac{1}{\Delta}\left(\frac{t^{q} \Delta_{3}+\Delta_{1} \Gamma(q+1)}{\Gamma(q+1)}\right)
\end{aligned}
$$




$$
\begin{aligned}
& \times\left[\int_{0}^{1} \frac{(1-s)^{q-1}}{\Gamma(q)}\right. \\
& \quad \times\left(\int_{0}^{s} \frac{(s-r)^{p-1}}{\Gamma(p)}\left(v_{n}(r)-v_{*}(s)\right) d r\right) d s \\
& \quad-\sum_{i=1}^{n} \alpha_{i} \\
& \quad \times \int_{0}^{\eta} \frac{(\eta-u)^{v_{i}-1}}{\Gamma\left(v_{i}\right)} \\
& \quad \times\left(\int_{0}^{u} \frac{(u-s)^{q-1}}{\Gamma(\mathrm{q})}\right. \\
& \quad \frac{1}{\Delta}\left(\frac{t^{q} \Delta_{2}+\Delta_{4} \Gamma(q+1)}{\Gamma(q+1)}\right) \\
& \left.\left.\left.\quad \times \sum_{i=1}^{n} \beta_{i}^{s} \frac{(s-r)^{p-1}}{\Gamma(p)}\left(v_{n}(r)-v_{*}(s)\right) d r\right\} d s\right) d u\right] \\
& \times \int_{0}^{\zeta} \frac{(\zeta-u)^{\mu_{i}-1}}{\Gamma\left(\mu_{i}\right)} \\
& \quad \times\left(\int_{0}^{u} \frac{(u-s)^{q-1}}{\Gamma(q)}\right. \\
& \left.\quad\left\{\int_{0}^{s} \frac{(s-r)^{p-1}}{\Gamma(p)}\left(v_{n}(r)-v_{*}(s)\right) d r\right\} d s\right) d u \|
\end{aligned}
$$

which tends to zero as $n \rightarrow \infty$.

Thus, it follows from Lemma 9 that $\Theta \circ S_{F}$ is a closed graph operator. Further, we have $h_{n}(t) \in \Theta\left(S_{F, x_{n}}\right)$. Since $x_{n} \rightarrow x_{*}$, it follows that

$h_{*}(t)$

$$
\begin{aligned}
=\int_{0}^{t} & \frac{(t-s)^{q-1}}{\Gamma(q)} \\
& \times\left(\int_{0}^{s} \frac{(s-r)^{p-1}}{\Gamma(p)} v_{*}(r) d r-\lambda x(s)\right) d s \\
- & \frac{1}{\Delta}\left(\frac{t^{q} \Delta_{3}+\Delta_{1} \Gamma(q+1)}{\Gamma(q+1)}\right) \\
\times\left[\int_{0}^{1} \frac{(1-s)^{q-1}}{\Gamma(q)}\right. & \quad \times\left(\int_{0}^{s} \frac{(s-r)^{p-1}}{\Gamma(p)} v_{*}(r) d r-\lambda x(s)\right) d s \\
& -\sum_{i=1}^{n} \alpha_{i} \\
& \times \int_{0}^{\eta} \frac{(\eta-u)^{v_{i}-1}}{\Gamma\left(v_{i}\right)}
\end{aligned}
$$

$$
\begin{gathered}
\times\left(\int_{0}^{u} \frac{(u-s)^{q-1}}{\Gamma(q)}\right. \\
\left.\left.\times\left\{\int_{0}^{s} \frac{(s-r)^{p-1}}{\Gamma(p)} v_{*}(r) d r-\lambda x(s)\right\} d s\right) d u\right] \\
-\frac{1}{\Delta}\left(\frac{t^{q} \Delta_{2}+\Delta_{4} \Gamma(q+1)}{\Gamma(q+1)}\right) \\
\times \sum_{i=1}^{n} \beta_{i} \\
\times \int_{0}^{\zeta} \frac{(\zeta-u)^{\mu_{i}-1}}{\Gamma\left(\mu_{i}\right)} \\
\times\left(\int_{0}^{u} \frac{(u-s)^{q-1}}{\Gamma(q)}\right. \\
\left.\times\left\{\int_{0}^{s} \frac{(s-r)^{p-1}}{\Gamma(p)} v_{*}(r) d r-\lambda x(s)\right\} d s\right) d u,
\end{gathered}
$$

for some $v_{*} \in S_{F, x_{*}}$.

Finally, we discuss a priori bounds on solutions. Let $x$ be a solution of (1). Then there exists $v \in L^{1}([0,1], \mathbb{R})$ with $v \in S_{F, x}$ such that, for $t \in[0,1]$, we have

$$
\begin{aligned}
& h(t) \\
& =\int_{0}^{t} \frac{(t-s)^{q-1}}{\Gamma(q)} \\
& \times\left(\int_{0}^{s} \frac{(s-r)^{p-1}}{\Gamma(p)} v(r) d r-\lambda x(s)\right) d s \\
& -\frac{1}{\Delta}\left(\frac{t^{q} \Delta_{3}+\Delta_{1} \Gamma(q+1)}{\Gamma(q+1)}\right) \\
& \times\left[\int_{0}^{1} \frac{(1-s)^{q-1}}{\Gamma(q)}\right. \\
& \times\left(\int_{0}^{s} \frac{(s-r)^{p-1}}{\Gamma(p)} v(r) d r-\lambda x(s)\right) d s \\
& -\sum_{i=1}^{n} \alpha_{i} \\
& \times \int_{0}^{\eta} \frac{(\eta-u)^{v_{i}-1}}{\Gamma\left(v_{i}\right)} \\
& \times\left(\int_{0}^{u} \frac{(u-s)^{q-1}}{\Gamma(q)}\right. \\
& \left.\left.\times\left\{\int_{0}^{s} \frac{(s-r)^{p-1}}{\Gamma(p)} v(r) d r-\lambda x(s)\right\} d s\right) d u\right] \\
& -\frac{1}{\Delta}\left(\frac{t^{q} \Delta_{2}+\Delta_{4} \Gamma(q+1)}{\Gamma(q+1)}\right) \\
& \times \sum_{i=1}^{n} \beta_{i}
\end{aligned}
$$




$$
\begin{aligned}
& \times \int_{0}^{\zeta} \frac{(\zeta-u)^{\mu_{i}-1}}{\Gamma\left(\mu_{i}\right)} \\
& \quad \times\left(\int_{0}^{u} \frac{(u-s)^{q-1}}{\Gamma(q)}\right. \\
& \left.\quad \times\left\{\int_{0}^{s} \frac{(s-r)^{p-1}}{\Gamma(p)} v(r) d r-\lambda x(s)\right\} d s\right) d u .
\end{aligned}
$$

Using the computations proving that $N(x)$ maps bounded sets into bounded sets and the notations (17), we have

$$
\begin{aligned}
&\|x\| \leq \psi(\|x\|)\|p\|_{L^{1}} \\
& \times\left\{\frac{1+A_{1}}{\Gamma(p+q+1)}\right. \\
&+A_{1} \sum_{i=1}^{n}\left|\alpha_{i}\right| \frac{\eta^{\nu_{i}+p+q}}{\Gamma\left(\nu_{i}+p+q+1\right)} \\
&\left.+A_{2} \sum_{i=1}^{n}\left|\beta_{i}\right| \frac{\zeta^{\mu_{i}+p+q}}{\Gamma\left(\mu_{i}+p+q+1\right)}\right\} \\
&+\|x\|\left\{\frac{|\lambda|\left(1+A_{1}\right)}{\Gamma(q+1)}\right.+|\lambda| A_{1} \sum_{i=1}^{n}\left|\alpha_{i}\right| \frac{\eta^{\nu_{i}+q}}{\Gamma\left(\nu_{i}+q+1\right)} \\
&\left.+A_{2} \sum_{i=1}^{n}\left|\beta_{i}\right| \frac{\zeta^{\mu_{i}+q}}{\Gamma\left(\mu_{i}+q+1\right)}\right\} \\
& \Omega \psi(\|x\|)\|p\|_{L^{1}}+\Psi\|x\| .
\end{aligned}
$$

Consequently

$$
\frac{\|x\|(1-\Psi)}{\psi(\|x\|)\|p\|_{L^{1}} \Omega} \leq 1 .
$$

In view of $\left(H_{3}\right)$, there exists $M$ such that $\|x\| \neq M$. Let us set

$$
U=\{x \in C([0,1], \mathbb{R}):\|x\|<M+1\} .
$$

Note that the operator $N: \bar{U} \rightarrow \mathscr{P}(C([0,1], \mathbb{R}))$ is upper semicontinuous and completely continuous. From the choice of $U$, there is no $x \in \partial U$ such that $x \in \mu N(x)$ for some $\mu \in(0,1)$. Consequently, by the nonlinear alternative of Leray-Schauder type [31], we deduce that $N$ has a fixed point $x \in \bar{U}$ which is a solution of the problem (1). This completes the proof.

3.2. The Lower Semicontinuous Case. Next, we study the case where $F$ is not necessarily convex valued. Our approach here is based on the nonlinear alternative of Leray-Schauder type combined with the selection theorem of Bressan and Colombo for lower semicontinuous maps with decomposable values.
Theorem 17. Assume that $\left(\mathrm{H}_{2}\right)-\left(\mathrm{H}_{3}\right)$ and the following conditions hold:

$\left(H_{4}\right) F:[0,1] \times \mathbb{R} \rightarrow \mathscr{P}(\mathbb{R})$ is a nonempty compact-valued multivalued map such that

(a) $(t, x) \mapsto F(t, x)$ is $\mathscr{L} \otimes \mathscr{B}$ measurable,

(b) $x \mapsto F(t, x)$ is lower semicontinuous for each $t \in$ $[0,1]$;

$\left(H_{5}\right)$ for each $\sigma>0$, there exists $\varphi_{\sigma} \in L^{1}\left([0,1], \mathbb{R}_{+}\right)$such that

$$
\begin{array}{r}
\|F(t, x)\|=\sup \{|y|: y \in F(t, x)\} \leq \varphi_{\sigma}(t) \\
\forall\|x\| \leq \sigma \text { and for a.e. } t \in[0,1] .
\end{array}
$$

Then the boundary value problem (1) has at least one solution on $[0,1]$.

Proof. It follows from $\left(\mathrm{H}_{4}\right)$ and $\left(\mathrm{H}_{5}\right)$ that $F$ is of l.s.c. type [35]. Then from Lemma 13, there exists a continuous function $f: C([0,1], \mathbb{R}) \rightarrow L^{1}([0,1], \mathbb{R})$ such that $f(x) \in \mathscr{F}(x)$ for all $x \in C([0,1], \mathbb{R})$.

Consider the problem

$$
\begin{gathered}
{ }^{c} D^{p}\left({ }^{c} D^{q}+\lambda\right) x(t)=F(x(t)), \quad 0<t<1, \\
x(0)=\sum_{i=1}^{n} \beta_{i}\left(I^{\mu_{i}} x\right)(\zeta), \\
x(1)=\sum_{i=1}^{n} \alpha_{i}\left(I^{\nu_{i}} x\right)(\eta), \quad 0<\zeta<\eta<1 .
\end{gathered}
$$

Observe that, if $x \in A C^{1}([0,1])$ is a solution of (43), then $x$ is a solution to the problem (1). In order to transform the problem (43) into a fixed point problem, we define the operator $\bar{N}$ as

$$
\begin{aligned}
& (\bar{N} x)(t) \\
& =\int_{0}^{t} \frac{(t-s)^{q-1}}{\Gamma(q)} \\
& \quad \times\left(\int_{0}^{s} \frac{(s-r)^{p-1}}{\Gamma(p)} f(x(r)) d r-\lambda x(s)\right) d s \\
& -\frac{1}{\Delta}\left(\frac{t^{q} \Delta_{3}+\Delta_{1} \Gamma(q+1)}{\Gamma(q+1)}\right)
\end{aligned}
$$




$$
\begin{aligned}
& \times\left[\int_{0}^{1} \frac{(1-s)^{q-1}}{\Gamma(q)}\right. \\
& \times\left(\int_{0}^{s} \frac{(s-r)^{p-1}}{\Gamma(p)} f(x(r)) d r-\lambda x(s)\right) d s \\
& -\sum_{i=1}^{n} \alpha_{i} \\
& \times \int_{0}^{\eta} \frac{(\eta-u)^{v_{i}-1}}{\Gamma\left(v_{i}\right)} \\
& \times\left(\int_{0}^{u} \frac{(u-s)^{q-1}}{\Gamma(q)}\right. \\
& \times\left\{\int_{0}^{s} \frac{(s-r)^{p-1}}{\Gamma(p)} f(x(r)) d r\right. \\
& -\lambda x(s)\} d s) d u] \\
& -\frac{1}{\Delta}\left(\frac{t^{q} \Delta_{2}+\Delta_{4} \Gamma(q+1)}{\Gamma(q+1)}\right) \\
& \times \sum_{i=1}^{n} \beta_{i} \\
& \times \int_{0}^{\zeta} \frac{(\zeta-u)^{\mu_{i}-1}}{\Gamma\left(\mu_{i}\right)} \\
& \times\left(\int_{0}^{u} \frac{(u-s)^{q-1}}{\Gamma(q)} v\right. \\
& \left.\times\left\{\int_{0}^{s} \frac{(s-r)^{p-1}}{\Gamma(p)} f(x(r)) d r-\lambda x(s)\right\} d s\right) d u .
\end{aligned}
$$

It can easily be shown that $\bar{N}$ is continuous and completely continuous. The remaining part of the proof is similar to that of Theorem 16. So we omit it. This completes the proof.

3.3. The Lipschitz Case. Now we prove the existence of solutions for the problem (1) with a nonconvex valued righthand side by applying a fixed point theorem for multivalued map due to Covitz and Nadler [34].

Theorem 18. Assume that the following conditions hold:

$\left(H_{6}\right) F:[0,1] \times \mathbb{R} \rightarrow \mathscr{P}_{c p}(\mathbb{R})$ is such that $F(\cdot, x):[0,1] \rightarrow$ $\mathscr{P}_{c p}(\mathbb{R})$ is measurable for each $x \in \mathbb{R}$;

$\left(H_{7}\right) H_{d}(F(t, x), F(t, \bar{x})) \leq m(t)|x-\bar{x}|$ for almost all $t \epsilon$ $[0,1]$ and $x, \bar{x} \in \mathbb{R}$ with $m \in C\left([0,1], \mathbb{R}^{+}\right)$and $d(0, F(t, 0)) \leq m(t)$ for almost all $t \in[0,1]$.
Then the boundary value problem (1) has at least one solution on $[0,1]$ if $\Omega\|m\|_{L^{1}}<1$, that is,

$$
\begin{aligned}
& \left\{\frac{1+A_{1}}{\Gamma(p+q+1)}+A_{1} \sum_{i=1}^{n}\left|\alpha_{i}\right| \frac{\eta^{v_{i}+p+q}}{\Gamma\left(v_{i}+p+q+1\right)}\right. \\
& \left.+A_{2} \sum_{i=1}^{n}\left|\beta_{i}\right| \frac{\zeta^{\mu_{i}+p+q}}{\Gamma\left(\mu_{i}+p+q+1\right)}\right\}\|m\|_{L^{1}}<1 .
\end{aligned}
$$

Proof. Observe that the set $S_{F, x}$ is nonempty for each $x \in$ $C([0,1], \mathbb{R})$ by the assumption $\left(H_{6}\right)$, so $F$ has a measurable selection (see Theorem III.6 [36]). Now we show that the operator $N$ satisfies the assumptions of Lemma 15. To show that $N(x) \in \mathscr{P}_{\mathrm{cl}}((C[0,1], \mathbb{R}))$ for each $x \in C([0,1], \mathbb{R})$, let $\left\{u_{n}\right\}_{n \geq 0} \in N(x)$ be such that $u_{n} \rightarrow u(n \rightarrow \infty)$ in $C([0,1], \mathbb{R})$. Then $u \in C([0,1], \mathbb{R})$, and there exists $v_{n} \in S_{F, x}$ such that, for each $t \in[0,1]$,

$$
\begin{aligned}
& u_{n}(t) \\
& =\int_{0}^{t} \frac{(t-s)^{q-1}}{\Gamma(q)} \\
& \times\left(\int_{0}^{s} \frac{(s-r)^{p-1}}{\Gamma(p)} v_{n}(r) d r-\lambda x(s)\right) d s \\
& -\frac{1}{\Delta}\left(\frac{t^{q} \Delta_{3}+\Delta_{1} \Gamma(q+1)}{\Gamma(q+1)}\right) \\
& \times\left[\int_{0}^{1} \frac{(1-s)^{q-1}}{\Gamma(q)}\right. \\
& \times\left(\int_{0}^{s} \frac{(s-r)^{p-1}}{\Gamma(p)} v_{n}(r) d r-\lambda x(s)\right) d s \\
& -\sum_{i=1}^{n} \alpha_{i} \\
& \times \int_{0}^{\eta} \frac{(\eta-u)^{v_{i}-1}}{\Gamma\left(v_{i}\right)} \\
& \times\left(\int_{0}^{u} \frac{(u-s)^{q-1}}{\Gamma(q)}\right. \\
& \left.\left.\times\left\{\int_{0}^{s} \frac{(s-r)^{p-1}}{\Gamma(p)} v_{n}(r) d r-\lambda x(s)\right\} d s\right) d u\right] \\
& -\frac{1}{\Delta}\left(\frac{t^{q} \Delta_{2}+\Delta_{4} \Gamma(q+1)}{\Gamma(q+1)}\right) \\
& \times \sum_{i=1}^{n} \beta_{i} \\
& \times \int_{0}^{\zeta} \frac{(\zeta-u)^{\mu_{i}-1}}{\Gamma\left(\mu_{i}\right)} \\
& \times\left(\int_{0}^{u} \frac{(u-s)^{q-1}}{\Gamma(q)}\right. \\
& \left.\times\left\{\int_{0}^{s} \frac{(s-r)^{p-1}}{\Gamma(p)} v_{n}(r) d r-\lambda x(s)\right\} d s\right) d u .
\end{aligned}
$$


As $F$ has compact values, we pass onto a subsequence to obtain that $v_{n}$ converges to $v$ in $L^{1}([0,1], \mathbb{R})$. Thus, $v \in S_{F, x}$ and, for each $t \in[0,1]$,

$$
\begin{aligned}
& u_{n}(t) \longrightarrow u(t) \\
& =\int_{0}^{t} \frac{(t-s)^{q-1}}{\Gamma(q)} \\
& \times\left(\int_{0}^{s} \frac{(s-r)^{p-1}}{\Gamma(p)} v(r) d r-\lambda x(s)\right) d s \\
& -\frac{1}{\Delta}\left(\frac{t^{q} \Delta_{3}+\Delta_{1} \Gamma(q+1)}{\Gamma(q+1)}\right) \\
& \times\left[\int_{0}^{1} \frac{(1-s)^{q-1}}{\Gamma(q)}\right. \\
& \times\left(\int_{0}^{s} \frac{(s-r)^{p-1}}{\Gamma(p)} v(r) d r-\lambda x(s)\right) d s \\
& -\sum_{i=1}^{n} \alpha_{i} \\
& \times \int_{0}^{\eta} \frac{(\eta-u)^{v_{i}-1}}{\Gamma\left(v_{i}\right)} \\
& \times\left(\int_{0}^{u} \frac{(u-s)^{q-1}}{\Gamma(q)}\right. \\
& \left.\left.\times\left\{\int_{0}^{s} \frac{(s-r)^{p-1}}{\Gamma(p)} v(r) d r-\lambda x(s)\right\} d s\right) d u\right] \\
& -\frac{1}{\Delta}\left(\frac{t^{q} \Delta_{2}+\Delta_{4} \Gamma(q+1)}{\Gamma(q+1)}\right) \\
& \times \sum_{i=1}^{n} \beta_{i} \\
& \times \int_{0}^{\zeta} \frac{(\zeta-u)^{\mu_{i}-1}}{\Gamma\left(\mu_{i}\right)} \\
& \times\left(\int_{0}^{u} \frac{(u-s)^{q-1}}{\Gamma(q)}\right. \\
& \left.\times\left\{\int_{0}^{s} \frac{(s-r)^{p-1}}{\Gamma(p)} v(r) d r-\lambda x(s)\right\} d s\right) d u .
\end{aligned}
$$

Hence, $u \in N(x)$.

Next we show that there exists $\gamma<1$ such that

$$
\begin{aligned}
& H_{d}(N(x), N(\bar{x})) \\
& \quad \leq \gamma\|x-\bar{x}\| \text { for each } x, \bar{x} \in C([0,1], \mathbb{R}) .
\end{aligned}
$$

Let $x, \bar{x} \in C([0,1], \mathbb{R})$ and $h_{1} \in N(x)$. Then there exists $v_{1}(t) \in F(t, x(t))$ such that, for each $t \in[0,1]$,

$h_{1}(t)$

$=\int_{0}^{t} \frac{(t-s)^{q-1}}{\Gamma(q)}$

$$
\times\left(\int_{0}^{s} \frac{(s-r)^{p-1}}{\Gamma(p)} v_{1}(r) d r-\lambda x(s)\right) d s
$$

$$
\begin{aligned}
& -\frac{1}{\Delta}\left(\frac{t^{q} \Delta_{3}+\Delta_{1} \Gamma(q+1)}{\Gamma(q+1)}\right) \\
& \times\left[\int_{0}^{1} \frac{(1-s)^{q-1}}{\Gamma(q)}\right. \\
& \times\left(\int_{0}^{s} \frac{(s-r)^{p-1}}{\Gamma(p)} v_{1}(r) d r-\lambda x(s)\right) d s \\
& -\sum_{i=1}^{n} \alpha_{i} \\
& \times \int_{0}^{\eta} \frac{(\eta-u)^{v_{i}-1}}{\Gamma\left(v_{i}\right)} \\
& \times\left(\int_{0}^{u} \frac{(u-s)^{q-1}}{\Gamma(q)}\right. \\
& \left.\left.\times\left\{\int_{0}^{s} \frac{(s-r)^{p-1}}{\Gamma(p)} v_{1}(r) d r-\lambda x(s)\right\} d s\right) d u\right] \\
& -\frac{1}{\Delta}\left(\frac{t^{q} \Delta_{2}+\Delta_{4} \Gamma(q+1)}{\Gamma(q+1)}\right) \\
& \times \sum_{i=1}^{n} \beta_{i} \\
& \times \int_{0}^{\zeta} \frac{(\zeta-u)^{\mu_{i}-1}}{\Gamma\left(\mu_{i}\right)} \\
& \times\left(\int_{0}^{u} \frac{(u-s)^{q-1}}{\Gamma(q)}\right. \\
& \left.\times\left\{\int_{0}^{s} \frac{(s-r)^{p-1}}{\Gamma(p)} v_{1}(r) d r-\lambda x(s)\right\} d s\right) d u .
\end{aligned}
$$

By $\left(H_{7}\right)$, we have

$$
H_{d}(F(t, x), F(t, \bar{x})) \leq m(t)|x(t)-\bar{x}(t)| .
$$

So, there exists $w(t) \in F(t, \bar{x}(t))$ such that

$$
\left|v_{1}(t)-w(t)\right| \leq m(t)|x(t)-\bar{x}(t)|, \quad t \in[0,1] .
$$

Define $U:[0,1] \rightarrow \mathscr{P}(\mathbb{R})$ by

$$
U(t)=\left\{w \in \mathbb{R}:\left|v_{1}(t)-w\right| \leq m(t)|x(t)-\bar{x}(t)|\right\} .
$$

Since the multivalued operator $U(t) \cap F(t, \bar{x}(t))$ is measurable (Proposition III.4 [36]), there exists a function $v_{2}(t)$ which is a measurable selection for $V$. So $v_{2}(t) \in F(t, \bar{x}(t))$, and for each $t \in[0,1]$, we have $\left|v_{1}(t)-v_{2}(t)\right| \leq m(t)|x(t)-\bar{x}(t)|$. 
For each $t \in[0,1]$, let us define

$h_{2}(t)$

$=\int_{0}^{t} \frac{(t-s)^{q-1}}{\Gamma(q)}$

$\times\left(\int_{0}^{s} \frac{(s-r)^{p-1}}{\Gamma(p)} v_{2}(r) d r-\lambda x(s)\right) d s$

$-\frac{1}{\Delta}\left(\frac{t^{q} \Delta_{3}+\Delta_{1} \Gamma(q+1)}{\Gamma(q+1)}\right)$

$\times\left[\int_{0}^{1} \frac{(1-s)^{q-1}}{\Gamma(q)}\right.$

$\times\left(\int_{0}^{s} \frac{(s-r)^{p-1}}{\Gamma(p)} v_{2}(r) d r-\lambda x(s)\right) d s$

$-\sum_{i=1}^{n} \alpha_{i}$

$\times \int_{0}^{\eta} \frac{(\eta-u)^{v_{i}-1}}{\Gamma\left(v_{i}\right)}$

$\times\left(\int_{0}^{u} \frac{(u-s)^{q-1}}{\Gamma(q)}\right.$

$\left.\left.\times\left\{\int_{0}^{s} \frac{(s-r)^{p-1}}{\Gamma(p)} v_{2}(r) d r-\lambda x(s)\right\} d s\right) d u\right]$

$-\frac{1}{\Delta}\left(\frac{t^{q} \Delta_{2}+\Delta_{4} \Gamma(q+1)}{\Gamma(q+1)}\right)$

$\times \sum_{i=1}^{n} \beta_{i}$

$\times \int_{0}^{\zeta} \frac{(\zeta-u)^{\mu_{i}-1}}{\Gamma\left(\mu_{i}\right)}$

$\times\left(\int_{0}^{u} \frac{(u-s)^{q-1}}{\Gamma(q)}\right.$

$\left.\times\left\{\int_{0}^{s} \frac{(s-r)^{p-1}}{\Gamma(p)} v_{2}(r) d r-\lambda x(s)\right\} d s\right) d u$.

Thus,

$\left|h_{1}(t)-h_{2}(t)\right|$

$=\int_{0}^{t} \frac{(t-s)^{q-1}}{\Gamma(q)}$

$\times\left(\int_{0}^{s} \frac{(s-r)^{p-1}}{\Gamma(p)}\left|v_{1}(r)-v_{2}(r)\right| d r\right) d s$

$$
-\frac{1}{\Delta}\left(\frac{t^{q} \Delta_{3}+\Delta_{1} \Gamma(q+1)}{\Gamma(q+1)}\right)
$$

$\times\left[\int_{0}^{1} \frac{(1-s)^{q-1}}{\Gamma(q)}\right.$

$\times\left(\int_{0}^{s} \frac{(s-r)^{p-1}}{\Gamma(p)}\left|v_{1}(r)-v_{2}(r)\right| d r\right) d s$

$-\sum_{i=1}^{n} \alpha_{i}$

$\times \int_{0}^{\eta} \frac{(\eta-u)^{v_{i}-1}}{\Gamma\left(v_{i}\right)}$

$\times\left(\int_{0}^{u} \frac{(u-s)^{q-1}}{\Gamma(q)}\right.$

$\left.\left.\times\left\{\int_{0}^{s} \frac{(s-r)^{p-1}}{\Gamma(p)}\left|v_{1}(r)-v_{2}(r)\right| d r\right\} d s\right) d u\right]$

$-\frac{1}{\Delta}\left(\frac{t^{q} \Delta_{2}+\Delta_{4} \Gamma(q+1)}{\Gamma(q+1)}\right)$

$\times \sum_{i=1}^{n} \beta_{i}$

$$
\times \int_{0}^{\zeta} \frac{(\zeta-u)^{\mu_{i}-1}}{\Gamma\left(\mu_{i}\right)}
$$$$
\times\left(\int_{0}^{u} \frac{(u-s)^{q-1}}{\Gamma(q)}\right.
$$

$\left.\times\left\{\int_{0}^{s} \frac{(s-r)^{p-1}}{\Gamma(p)}\left|v_{1}(r)-v_{2}(r)\right| d r\right\} d s\right) d u$.

Hence,

$$
\left\|h_{1}-h_{2}\right\| \leq\left\{\frac{1+A_{1}}{\Gamma(p+q+1)}\right.
$$

$$
+A_{1} \sum_{i=1}^{n}\left|\alpha_{i}\right| \frac{\eta^{\nu_{i}+p+q}}{\Gamma\left(v_{i}+p+q+1\right)}
$$

$$
\left.+A_{2} \sum_{i=1}^{n}\left|\beta_{i}\right| \frac{\zeta^{\mu_{i}+p+q}}{\Gamma\left(\mu_{i}+p+q+1\right)}\right\}
$$

$\times\|m\|_{L^{1}}\|x-\bar{x}\|$. 
Analogously, interchanging the roles of $x$ and $\bar{x}$, we obtain

$$
\begin{aligned}
H_{d}(N(x), N(\bar{x})) \leq & \gamma\|x-\bar{x}\| \\
\leq & \left\{\frac{1+A_{1}}{\Gamma(p+q+1)}\right. \\
& +A_{1} \sum_{i=1}^{n}\left|\alpha_{i}\right| \frac{\eta^{\nu_{i}+p+q}}{\Gamma\left(\nu_{i}+p+q+1\right)} \\
& \left.+A_{2} \sum_{i=1}^{n}\left|\beta_{i}\right| \frac{\zeta^{\mu_{i}+p+q}}{\Gamma\left(\mu_{i}+p+q+1\right)}\right\} \\
& \times\|m\|_{L^{1}}\|x-\bar{x}\| .
\end{aligned}
$$

Since $N$ is a contraction, it follows from Lemma 15 that $N$ has a fixed point $x$ which is a solution of (1). This completes the proof.

Remark 19. It is important to note that several new interesting special results of the present work can be obtained by fixing the parameters involved in the given problem. Some of these results are listed in the following.

(i) Our results correspond to the multivalued extension of the Dirichlet problem considered in [23] for $\beta_{i}=$ $0=\alpha_{i}, i=1,2, \ldots, n$.

(ii) In case we take $\beta_{i}=0, i=1,2, \ldots, n$, we obtain the results for Langevin fractional differential inclusions with the three-point integral boundary conditions of the following form:

$$
x(0)=0, \quad x(1)=\sum_{i=1}^{n} \alpha_{i}\left(I^{v_{i}} x\right)(\eta) .
$$

(iii) By taking $\beta_{i}=0=\alpha_{i}, i=2,3, \ldots, n$, and $\nu_{1}=1=\mu_{1}$, we get the results for Langevin fractional differential inclusions with four-point nonlocal integral boundary conditions of the following type:

$$
x(0)=\beta_{1} \int_{0}^{\zeta} x(s) d s, \quad x(1)=\alpha_{1} \int_{0}^{\eta} x(s) d s .
$$

Note that we obtain the typical integral boundary condition in the limit $\zeta, \eta \rightarrow 1^{-}$.

Example 20. Consider the problem

$$
\begin{aligned}
{ }^{c} D^{2 / 3}\left({ }^{c} D^{4 / 5}+\frac{1}{10}\right) x(t) \in F(t, x(t)), \quad 0<t<1, \\
x(0)=\frac{1}{2}\left(I^{1 / 2} x\right)\left(\frac{1}{4}\right)-\frac{1}{3}\left(I^{3 / 4} x\right)\left(\frac{1}{4}\right) \\
+\frac{1}{4}\left(I^{3 / 2} x\right)\left(\frac{1}{4}\right)-\frac{1}{5}\left(I^{4 / 3} x\right)\left(\frac{1}{4}\right) \\
x(1)=\frac{1}{2}\left(I^{1 / 4} x\right)\left(\frac{1}{3}\right)-\frac{2}{3}\left(I^{3 / 4} x\right)\left(\frac{1}{3}\right) \\
+\frac{3}{4}\left(I^{5 / 4} x\right)\left(\frac{1}{3}\right)-\frac{4}{5}\left(I^{7 / 4} x\right)\left(\frac{1}{3}\right)
\end{aligned}
$$

where $F:[0,1] \times \mathbb{R} \rightarrow \mathscr{P}(\mathbb{R})$ is a multivalued map given by

$$
x \longrightarrow F(t, x)=\left[\frac{1}{4} \frac{x^{5}}{x^{5}+3}+\frac{1}{8}(t+1), \frac{1}{4} \sin x+\frac{1}{4}(t+1)\right] \text {. }
$$

For $f \in F$, we have

$$
|f| \leq \max \left[\frac{1}{4} \frac{x^{5}}{x^{5}+3}+\frac{1}{8}(t+1), \frac{1}{4} \sin x+\frac{1}{4}(t+1)\right] \leq \frac{3}{4} .
$$

Here $p=2 / 3, q=4 / 5, \lambda=1 / 10, \eta=1 / 3, n=4, \alpha_{1}=$ $1 / 2, \alpha_{2}=-2 / 3, \alpha_{3}=3 / 4, \alpha_{4}=-4 / 5, \nu_{1}=1 / 4, v_{2}=$ $3 / 4, v_{3}=5 / 4, v_{4}=7 / 4, \zeta=1 / 4, \beta_{1}=1 / 2, \beta_{2}=-1 / 3, \beta_{3}=$ $1 / 4, \beta_{4}=-1 / 5, \mu_{1}=1 / 2, \mu_{2}=3 / 4, \mu_{3}=3 / 2$, and $\mu_{4}=4 / 3$. Clearly,

$$
\begin{aligned}
& \|F(t, x)\|_{\mathscr{P}} \\
& \quad:=\sup \{|y|: y \in F(t, x)\} \leq p(t) \psi(\|x\|), \quad x \in \mathbb{R},
\end{aligned}
$$

with $p(t)=1, \psi(\|x\|)=3 / 4$. Using the given data, it is found that

$$
\begin{aligned}
& \Delta_{1}=0.126506, \Delta_{2}=0.804143, \\
& \Delta_{3}=0.849082, \Delta_{4}=-0.983656, \\
& \Delta=0.936934, A_{1}=1.108019, \\
& A_{2}=1.971367, \quad \Omega=1.764724, \\
& \Psi=0.47047 .
\end{aligned}
$$

Thus,

$$
M>\frac{\psi(M)\|p\|_{L^{1}} \Omega}{1-\Psi} \approx 2.499467 .
$$

Clearly, all the conditions of Theorem 16 are satisfied. So there exists at least one solution of the problem (59) on $[0,1]$.

Example 21. Consider the fractional inclusion boundary value problem (59) with $F:[0,1] \times \mathbb{R} \rightarrow \mathscr{P}(\mathbb{R})$ given by

$$
F(t, x)=\left[0, \frac{\sin x}{(2+t)^{2}}+\frac{1}{12}\right] \text {. }
$$

Then, we have

$$
\begin{gathered}
\sup \{|u|: u \in F(t, x)\} \leq \frac{1}{12}+\frac{1}{(2+t)^{2}}, \\
H_{d}(F(t, x), F(t, \bar{x})) \leq m(t)|x-\bar{x}|
\end{gathered}
$$

where $m(t)=1 /(2+t)^{2}$. With $\|m\|_{L^{1}}=1 / 6$ and $\Omega=1.764724$ (from Example 20), it is found that

$$
\gamma=\Omega\|m\|_{L^{1}} \approx 0.294121<1 .
$$

Since all the conditions of Theorem 18 are satisfied, therefore the problem (59) with $F$ given by (65) has at least one solution on $[0,1]$. 


\section{Acknowledgments}

The authors thank the referees for their useful comments. This work was partially supported by Deanship of Scientific Research (DSR), King Abdulaziz University, Jeddah, Saudi Arabia.

\section{References}

[1] S. G. Samko, A. A. Kilbas, and O. I. Marichev, Fractional Integrals and Derivatives, Gordon and Breach Science Publishers, Yverdon, Switzerland, 1993.

[2] I. Podlubny, Fractional Differential Equations, vol. 198 of Mathematics in Science and Engineering, Academic Press, San Diego, CA, USA, 1999.

[3] A. A. Kilbas, H. M. Srivastava, and J. J. Trujillo, Theory and Applications of Fractional Differential Equations, vol. 204 of North-Holland Mathematics Studies, Elsevier Science, Amsterdam, The Netherlands, 2006.

[4] J. Sabatier, O. P. Agrawal, and J. A. T. Machado, Eds., Advances in Fractional Calculus: Theoretical Developments and Applications in Physics and Engineering, Springer, Dordrecht, The Netherlands, 2007.

[5] V. Lakshmikantham, S. Leela, and J. Vasundhara Devi, Theory of Fractional Dynamic Systems, Cambridge Academic Publishers, Cambridge, UK, 2009.

[6] D. Baleanu, A. K. Golmankhaneh, and A. K. Golmankhaneh, "Fractional Nambu mechanics," International Journal of Theoretical Physics, vol. 48, no. 4, pp. 1044-1052, 2009.

[7] B. Ahmad and S. K. Ntouyas, "Nonlinear fractional differential equations and inclusions of arbitrary order and multi-strip boundary conditions," Electronic Journal of Differential Equations, vol. 2012, no. 98, pp. 1-22, 2012.

[8] Nyamoradi N. and M. Javidi, "Existence of multiple positive solutions for fractional differential inclusion with $m$-point boundary conditions and two fractional orders," Electronic Journal of Differential Equations, vol. 2012, no. 187, pp. 1-26, 2012.

[9] B. Ahmad and J. J. Nieto, "Sequential fractional differential equations with three-point boundary conditions," Computers \& Mathematics with Applications, vol. 64, no. 10, pp. 3046-3052, 2012.

[10] M. R. Ubriaco, "Entropies based on fractional calculus," Physics Letters A, vol. 373, no. 30, pp. 2516-2519, 2009.

[11] J. Henderson and A. Ouahab, "Fractional functional differential inclusions with finite delay," Nonlinear Analysis. Theory, Methods \& Applications, vol. 70, no. 5, pp. 2091-2105, 2009.

[12] R. P. Agarwal, M. Belmekki, and M. Benchohra, "A survey on semilinear differential equations and inclusions involving Riemann-Liouville fractional derivative," Advances in Difference Equations, Article ID 981728, p. 47, 2009.

[13] Y.-K. Chang and J. J. Nieto, "Some new existence results for fractional differential inclusions with boundary conditions," Mathematical and Computer Modelling, vol. 49, no. 3-4, pp. 605609, 2009.

[14] S. Hamani, M. Benchohra, and J. R. Graef, "Existence results for boundary-value problems with nonlinear fractional differential inclusions and integral conditions," Electronic Journal of Differential Equations, vol. 2010, no. 20, pp. 1-16, 2010.

[15] A. Cernea, "On the existence of solutions for nonconvex fractional hyperbolic differential inclusions," Communications in Mathematical Analysis, vol. 9, no. 1, pp. 109-120, 2010.
[16] B. Ahmad and S. K. Ntouyas, "Some existence results for boundary value problems of fractional differential inclusions with non-separated boundary conditions," Electronic Journal of Qualitative Theory of Differential Equations, no. 71, pp. 1-17, 2010.

[17] W. Sudsutad and J. Tariboon, "Existence results of fractional integro-differential equations with m-point multi-term fractional order integral boundary conditions," Boundary Value Problems, vol. 2012, article 94, 2012.

[18] W. T. Coffey, Y. P. Kalmykov, and J. T. Waldron, The Langevin Equation, vol. 14 of World Scientific Series in Contemporary Chemical Physics, World Scientific Publishing, River Edge, NJ, USA, 2nd edition, 2004.

[19] S. C. Lim, M. Li, and L. P. Teo, "Langevin equation with two fractional orders," Physics Letters A, vol. 372, no. 42, pp. 63096320, 2008.

[20] S. C. Lim and L. P. Teo, "The fractional oscillator process with two indices," Journal of Physics A, vol. 42, no. 6, Article ID 065208, 2009.

[21] M. Uranagase and T. Munakata, "Generalized Langevin equation revisited: mechanical random force and self-consistent structure," Journal of Physics A, vol. 43, no. 45, Article ID 455003, 2010.

[22] S. I. Denisov, H. Kantz, and P. Hänggi, "Langevin equation with super-heavy-tailed noise," Journal of Physics A, vol. 43, no. 28, Article ID 285004, 2010.

[23] B. Ahmad and J. J. Nieto, "Solvability of nonlinear Langevin equation involving two fractional orders with Dirichlet boundary conditions," International Journal of Differential Equations, Article ID 649486, 10 pages, 2010.

[24] A. Lozinski, R. G. Owens, and T. N. Phillips, “The Langevin and Fokker-Planck equations in polymer rheology," Handbook of Numerical Analysis, vol. 16, pp. 211-303, 2011.

[25] L. Lizana, T. Ambjörnsson, A. Taloni, E. Barkai, and M. A. Lomholt, "Foundation of fractional Langevin equation: harmonization of a many-body problem," Physical Review E, vol. 81, no. 5,8 pages, 2010 .

[26] B. Ahmad, J. J. Nieto, A. Alsaedi, and M. El-Shahed, "A study of nonlinear Langevin equation involving two fractional orders in different intervals," Nonlinear Analysis. Real World Applications, vol. 13, no. 2, pp. 599-606, 2012.

[27] K. Deimling, Multivalued Differential Equations, vol. 1 of de Gruyter Series in Nonlinear Analysis and Applications, Walter de Gruyter, Berlin, Germany, 1992.

[28] S. Hu and N. S. Papageorgiou, Handbook of Multivalued Analysis, vol. I of Mathematics and its Applications, Kluwer Academic Publishers, Dordrecht, The Netherlands, 1997.

[29] G. V. Smirnov, Introduction to the Theory of Differential Inclusions, vol. 41 of Graduate Studies in Mathematics, American Mathematical Society, Providence, RI, USA, 2002.

[30] A. Lasota and Z. Opial, "An application of the Kakutani-Ky Fan theorem in the theory of ordinary differential equations," Bulletin de l'Académie Polonaise des Sciences. Série des Sciences Mathématiques, vol. 13, pp. 781-786, 1965.

[31] A. Granas and J. Dugundji, Fixed Point Theory, Springer Monographs in Mathematics, Springer, New York, NY, USA, 2005.

[32] A. Bressan and G. Colombo, "Extensions and selections of maps with decomposable values," Studia Mathematica, vol. 90, no. 1, pp. 69-86, 1988. 
[33] M. Kisielewicz, Differential Inclusions and Optimal Control, vol. 44 of Mathematics and its Applications (East European Series), Kluwer Academic Publishers Group, Dordrecht, The Netherlands, 1991.

[34] H. Covitz and S. B. Nadler Jr., "Multi-valued contraction mappings in generalized metric spaces," Israel Journal of Mathematics, vol. 8, pp. 5-11, 1970.

[35] M. Frigon, "Théorèmes d'existence de solutions d'inclusions différentielles," in Topological Methods in Differential Equations and Inclusions, A. Granas and M. Frigon, Eds., vol. 472 of NATO ASI Series C, pp. 51-87, Kluwer Academic, Dordrecht, The Netherlands, 1995.

[36] C. Castaing and M. Valadier, Convex Analysis and Measurable Multifunctions, vol. 580 of Lecture Notes in Mathematics, Springer, Berlin, Germany, 1977. 


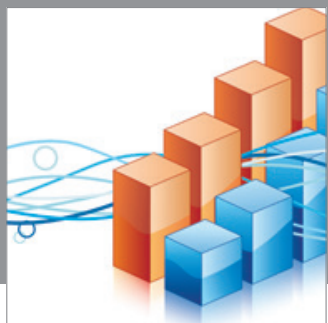

Advances in

Operations Research

mansans

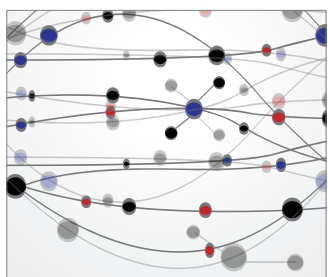

The Scientific World Journal
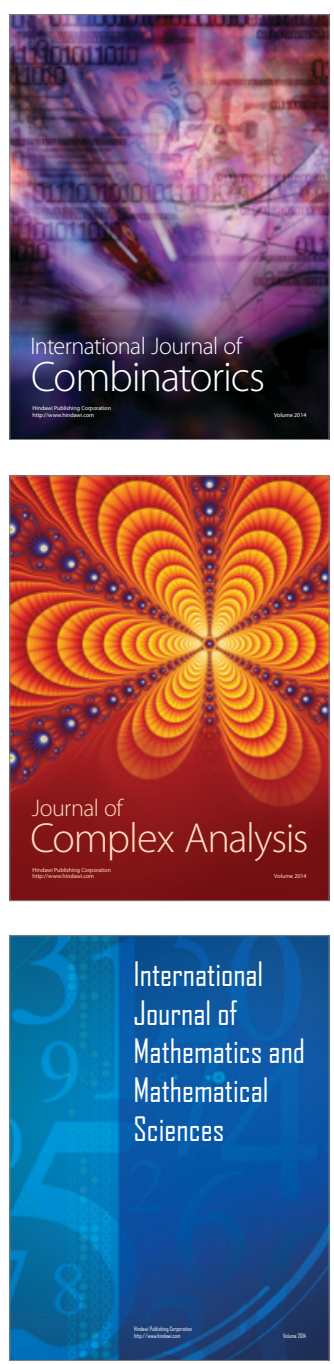
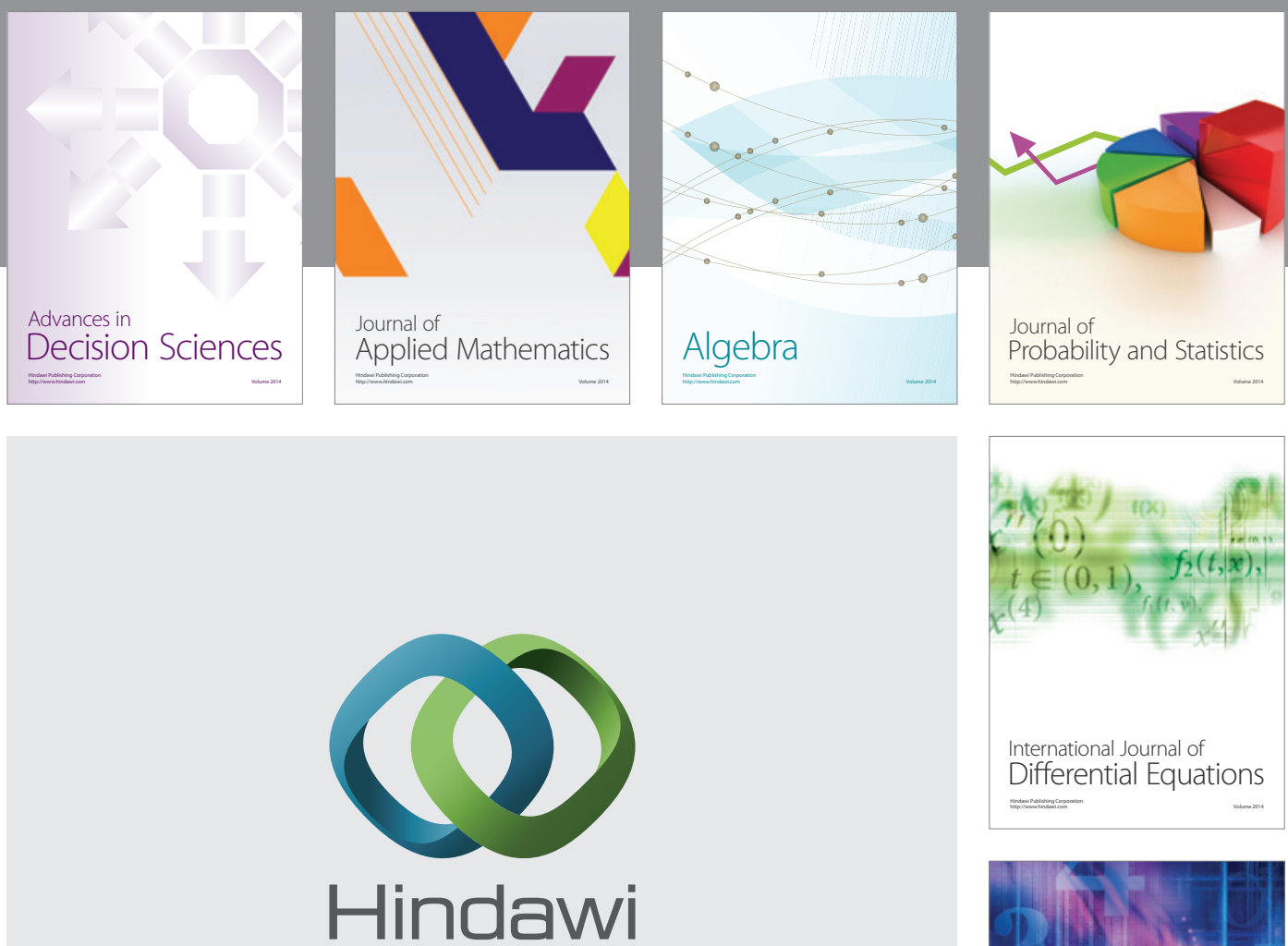

Submit your manuscripts at http://www.hindawi.com
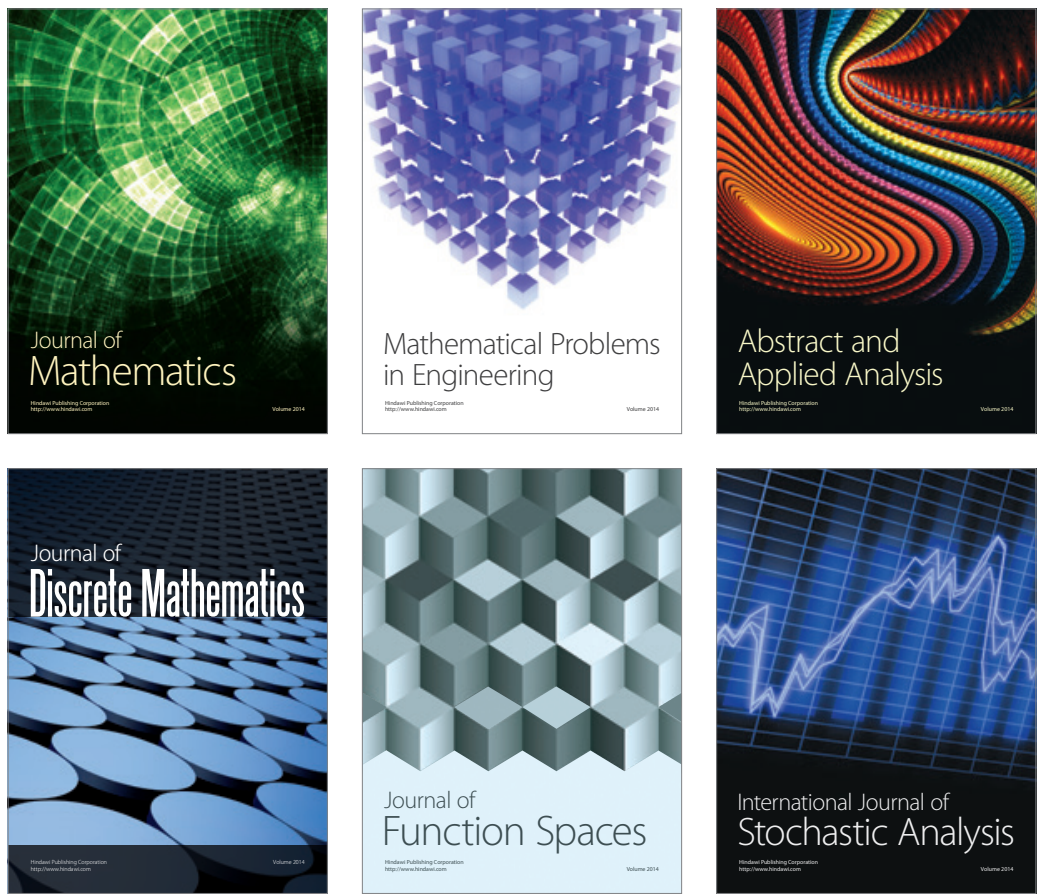

Journal of

Function Spaces

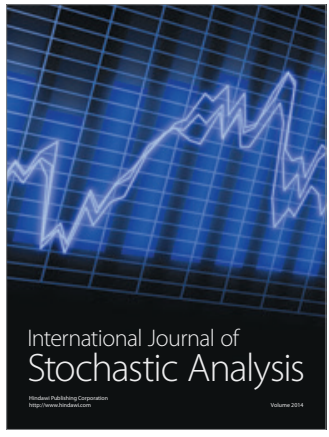

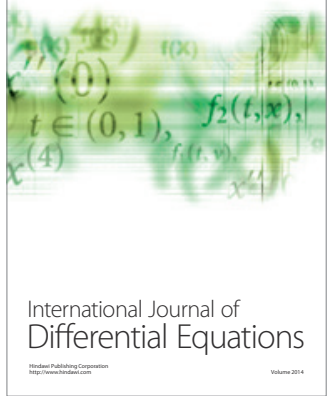
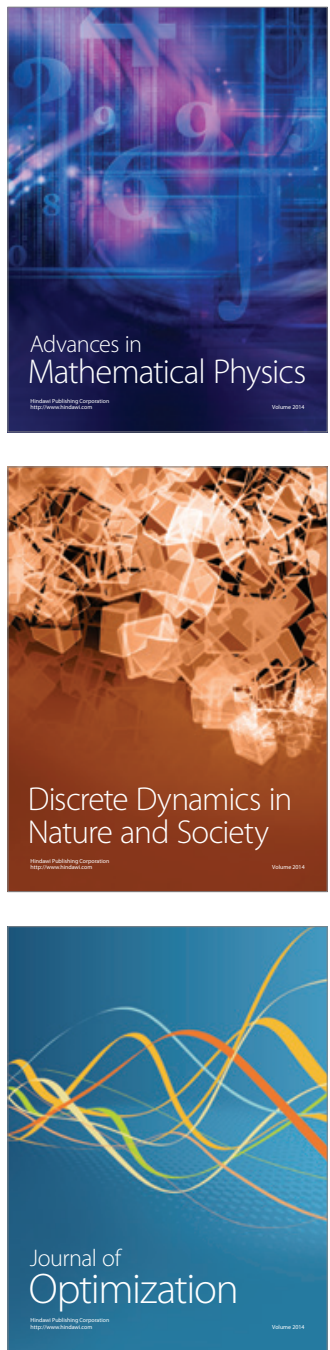